\title{
HIGH-ORDER ESSENTIALLY NON-OSCILLATORY SCHEME FOR VISCOELASTICITY WITH FADING MEMORY
}

\author{
BY \\ CHI-WANG SHU AND YANNI ZENG \\ Division of Applied Mathematics, Brown University, Providence, RI
}

\begin{abstract}
In this paper we describe the application of high-order essentially nonoscillatory (ENO) finite difference schemes to the viscoelastic model with fading memory. ENO schemes can capture shocks as well as various smooth structures in the solution to a high-order accuracy without spurious numerical oscillations. We first verify the stability and resolution of the scheme. We apply the scheme to a nonlinear problem with a known smooth solution and check the order of accuracy. Then we apply the scheme to a linear problem with initial discontinuities. Discontinuity locations and strengths in the solutions of such problems can be found explicitly by making use of a pointwise estimate obtained in this paper for the Green's function of the equations, which contains two Dirac $\delta$-functions decaying exponentially. We check the resolution of the discontinuities by the scheme. After verifying that the scheme is indeed high-order accurate, produces sharp, non-oscillatory shocks with the correct location and strength, we then proceed in applying it to the nonlinear case with discontinuous or smooth initial conditions, and study the local properties (in time) as well as the long time behavior of the solutions. We conclude that the ENO scheme is a robust, accurate numerical tool to supplement theoretical analysis to study such equations with memory terms. It should also provide an efficient and reliable practical tool when such equations must be solved numerically in applications.
\end{abstract}

Received September 28, 1994.

1991 Mathematics Subject Classification. Primary 35A40, 65M06.

Key words and phrases. Viscoelasticity, fading memory, essentially non-oscillatory schemes, high-order accuracy.

Research supported by ONR grant N00014-92-J-1481. Additional support for the first author was also provided by ARO grant DAAL03-91-G-0123 and DAAH04-94-G-0205, NSF grant DMS-9211820, and AFOSR grant 93-0090. Additional support for the second author was also provided by ARO grant DAAH04-93-G-0125 and NSF grant DMS-9307928. Computation supported by NAS.

Current address of Yanni Zeng: Department of Applied Mathematics and Statistics, SUNY at Stony Brook, Stony Brook, NY 11794. 
1. Introduction. In this paper we are interested in solving the following Cauchy problem:

$$
\begin{gathered}
\left\{\begin{array}{l}
u_{t}-v_{x}=0, \\
v_{t}-\sigma_{x}=g, \quad x \in \mathcal{R}, t>0,
\end{array}\right. \\
u(x, 0)=u_{0}(x), \quad v(x, 0)=v_{0}(x), \quad x \in \mathcal{R},
\end{gathered}
$$

where $g(x, t)$ is a given function, and the function $\sigma$ is determined by $u$ and its past history through the constitutive law

$$
\sigma(x, t)=\phi(u(x, t))+\int_{0}^{t} a^{\prime}(t-\tau) \psi(u(x, \tau)) d \tau
$$

for given functions $\phi(u), \psi(u)$, and $a(t)$ with derivative $a^{\prime}$. We assume that $\phi, \psi$, and $a$ are smooth, and

$$
\phi^{\prime}(u)>0, \quad \psi^{\prime}(u)>0, \quad \phi^{\prime}(u)-a(0) \psi^{\prime}(u)>0 .
$$

System (1.1) is a model for the motion of an unbounded, homogeneous, viscoelastic bar with fading memory. The functions $u, v, \sigma$, and $g$ represent the strain deformation, velocity, stress, and body force, respectively. In the constitutive law (1.3), $\phi$ and $\psi$ are material functions, while the kernel $a$ has the following form in the most important physical application:

$$
a(t)=\sum_{j=1}^{N} c_{j} e^{-\mu_{j} t}, \quad t \geq 0,
$$

with $c_{j}, \mu_{j}>0, j=1, \ldots, N$.

System (1.1), (1.3) is hyperbolic under assumption (1.4). Particularly, it reduces to the $p$-system if $a(t)=1, t \in[0, \infty)$, in (1.3). In this case, taking $g \equiv 0$, it is typical that singularities develop in a finite time, even if the initial data are smooth, which propagate as shock waves, [8]. If $a$ is of the form (1.5), however, the memory term in (1.1) induces a weak dissipative mechanism. An interesting problem is how this dissipative mechanism competes with the hyperbolic character of the system. If the initial data $u_{0}$ and $v_{0}$ and the body force $g$ are smooth and small, the dissipation is strong enough to prevent the breakdown of smooth solutions. It has been shown that in this case the initial value problem (1.1)-(1.3) has a unique globally defined classical solution, which decays to the equilibrium as $t \rightarrow \infty$; see [6], [3], and [20]; also see [12] and [4] for the initial boundary value problems. Large-time behavior of the solution has been studied in [21]. If the initial data are smooth but large, solutions of (1.1)-(1.3) may develop singularities in their derivatives in finite time; see [2] and [14]. Numerical evidence also suggests that shock waves may develop in this case; see [13] and Example 3 in Sec. 4 below. If the initial data are not smooth, one has to consider weak solutions. In the case $\phi=\psi$ and $u_{0}, v_{0} \in L^{\infty}(\mathcal{R}) \cap L^{2}(\mathcal{R})$, Nohel, Rogers, and Tzavaras, [15], have proved the existence of the weak solutions of (1.1)-(1.3) for more general kernel $a$, using the method of compensated compactness. However, there is no physical motivation for the restriction $\phi=\psi$. It is also interesting to consider solutions with initial data not in $L^{2}(\mathcal{R})$, e.g., solutions with Riemann data. For more general systems and other 
initial data, unfortunately, there is no theory for weak solutions at all. In Sec. 3 below, we obtain a nice point-wise estimate for the Green's function of the linearized system. The estimate contains two Dirac $\delta$-functions exponentially decaying in time. For linear systems, these $\delta$-functions provide us with all the information, the locations and the amplitudes, of the discontinuities in the solution generated by the initial discontinuities. For nonlinear systems, the $\delta$-functions suggest that any initial discontinuity propagates into the solution for all time, and decays exponentially if the initial jump is small.

Since the system (1.1)-(1.3) may have discontinuous solutions, traditional high-order numerical methods may not be stable. In [13] a second-order Lax-Wendroff method is proposed to solve this system, and stability and convergence are proven with the assumption that the solution stays smooth. When this assumption is violated, the numerical solution shows signs of instability such as over/under shoots and oscillations near the discontinuity, which may pollute into smooth regions for later time (see the pictures in [13]). Although artificial viscosity may reduce such oscillations to a certain extent, modern nonlinear "high resolution" schemes [9] are more adequate if one wants to completely remove the oscillations while still keeping the uniform high-order accuracy in the smooth regions.

In this paper we discuss the application of ENO (Essentially Non-Oscillatory) schemes for solving the system (1.1)-(1.3). ENO schemes, first developed by Harten, Engquist, Osher, and Chakravarthy [5] in the cell averaged formulation and later by Shu and Osher [16], [17] in a simplified formulation using only point values and TVD (total variation diminishing) Runge-Kutta type stepping, are based on an adaptive stencil interpolation idea to automatically interpolate in a locally smoothest region, thus avoiding crossing discontinuities or high gradient regions as much as possible, while still maintaining highorder accuracy. We will review ENO schemes and describe their application to the system (1.1)-(1.3) in Sec. 2. In Sec. 3 we will develop some mathematical properties of the system (1.1)-(1.3) and its linearized version. Section 4 contains numerical examples, first to validate the ENO scheme, then to use it as a tool to study local (in time) as well as long-time behavior of the solutions to (1.1)-(1.3). Concluding remarks are given in Sec. 5 .

2. ENO schemes. In this section we shall first briefly review the point-wise ENO (Essentially Non-Oscillatory) high-order finite difference method for a general hyperbolic conservation law with shocks. More details can be found in [5] and [17]; see also [16], [18], [19], and [1]. We shall then describe the application of ENO schemes to the system (1.1)-(1.3).

The first ENO schemes, developed by Harten, Engquist, Osher, and Chakravarthy [5], are in the cell-averaged formulation, which is rather expensive for multi space dimensions. [16], [17], [18], and [19] describe the point-wise ENO schemes. In two space dimensions, a point-wise ENO is about four times as fast as a cell-averaged one. In three dimensions this factor is about nine. We shall use point value ENO schemes in this paper.

We first explain how a scalar one-dimensional conservation law

$$
u_{t}+f(u)_{x}=0
$$


is solved by point value ENO schemes. Leaving, for the time being, the time variable $t$ undiscretized, we have the conservation scheme:

$$
\frac{d u_{j}}{d t}=-\frac{1}{\Delta x}\left(\hat{f}_{j+\frac{1}{2}}-\hat{f}_{j-\frac{1}{2}}\right)
$$

where $u_{j}(t)$ approximates $u\left(x_{j}, t\right)$ and $\hat{f}_{j+\frac{1}{2}}$ is the numerical flux function. This scheme is $r$ th-order accurate:

$$
\frac{1}{\Delta x}\left(\hat{f}_{j+\frac{1}{2}}-\hat{f}_{j-\frac{1}{2}}\right)=\left.f(u)_{x}\right|_{x=x_{j}}+O\left(\Delta x^{r}\right)
$$

if the numerical flux $\hat{f}_{j+\frac{1}{2}}$ satisfies

$$
\hat{f}_{j+\frac{1}{2}}=h\left(x_{j+\frac{1}{2}}\right)+O\left(\Delta x^{r+1}\right),
$$

or just $O\left(\Delta x^{r}\right)$ if this term is smooth, where the function $h(x)$ is defined implicitly by

$$
f(u(x))=\frac{1}{\Delta x} \int_{x-\frac{\Delta x}{2}}^{x+\frac{\Delta x}{2}} h(\xi) d \xi
$$

where we have suppressed the time variable $t$. The simple proof of this fact is given in [17]. Even if $h(x)$ is only defined implicitly by (2.5), it is easy to approximate the primitive of $h(x)$ defined by $H(x)=\int_{-\infty}^{x} h(\xi) d \xi$, since

$$
H\left(x_{j+\frac{1}{2}}\right)=\Delta x \sum_{k=-\infty}^{j} f\left(u\left(x_{k}\right)\right)
$$

and $f\left(u\left(x_{k}\right)\right)$ are explicitly known. Notice that the lower limit $-\infty$ is irrelevant and can be replaced by any fixed index.

The procedure to compute the flux $\hat{f}_{j+\frac{1}{2}}$ is thus summarized as follows: Given the point values $u\left(x_{k}\right)$, hence $f\left(u\left(x_{k}\right)\right)$, one obtains $H\left(x_{j+\frac{1}{2}}\right)$ using (2.6), and then builds a polynomial $P_{j+\frac{1}{2}}(x)$, for each $j$, of degree $r+1$ that interpolates $H\left(x_{k+\frac{1}{2}}\right)$ at $r+2$ consecutive grid points including $x_{j+\frac{1}{2}}$. Notice that there are $r+2$ choices for the stencil of $P_{j+\frac{1}{2}}(x)$, since the left-most point in the stencil can be anywhere between $x_{j-r-\frac{1}{2}}$ and $x_{j+\frac{1}{2}}$. For stability considerations (upwinding), we require the stencil to contain two points $\left\{x_{j-\frac{1}{2}}, x_{j+\frac{1}{2}}\right\}$ or $\left\{x_{j+\frac{1}{2}}, x_{j+\frac{3}{2}}\right\}$ depending on the local wind direction. This leaves us with $r+1$ possibilities for the stencil. For all those choices, approximation theory of polynomial interpolation tells us that

$$
\frac{d}{d x} P_{j+\frac{1}{2}}\left(x_{j+\frac{1}{2}}\right)=\frac{d}{d x} H\left(x_{j+\frac{1}{2}}\right)+O\left(\Delta x^{r+1}\right)
$$

provided that the function $H(x)$ is smooth (at least $C^{r+2}$ ) in the stencil of this interpolating polynomial $P_{j+\frac{1}{2}}(x)$. One thus only needs to take $\hat{f}_{j+\frac{1}{2}}=\frac{d}{d x} P_{j+\frac{1}{2}}\left(x_{j+\frac{1}{2}}\right)$ to guarantee $r$ th-order accuracy (2.4), if one uses a stencil in which $H(x)$, or equivalently $f(u(x))$, is smooth.

If the function $f(u(x))$ is globally smooth, one can of course choose any stencil for $P_{j+\frac{1}{2}}(x)$ to claim the required accuracy (2.4). One then is motivated to choose the stencil with the smallest coefficient in the error term $O\left(\Delta x^{r+1}\right)$, leading naturally to the usual central stencil (e.g., $\left\{x_{j-\frac{1}{2}}, x_{j+\frac{1}{2}}, x_{j+\frac{3}{2}}\right\}$ for the case $r=1$ ) for all $j$. This is the basis for the traditional "fixed stencil" schemes, which are linear when applied to linear problems 
and a Fourier stability analysis can be applied to obtain the stability and convergence for either linear or nonlinear PDE's with smooth solutions. This is, for example, the procedure adopted in [13].

However, if the function $f(u(x))$, hence $H(x)$, is only piecewise smooth with shocks in between, the fixed stencil approximation will cross the shock to build a high-order polynomial interpolation. Not only is the accuracy totally lost in such cases, the approximation also shows highly oscillatory results. This is why fixed stencil schemes, whether they are linearly stable or not, often give poor results in the presence of shocks.

ENO interpolation tries to overcome this difficulty by adaptively choosing its stencil so that at each $x_{j+\frac{1}{2}}$, the smoothest (in some sense) stencil out of all the $r+1$ possibilities is used. The basic idea is as follows: suppose the order of $P_{j+\frac{1}{2}}(x)$ is one and we must use $x_{j+\frac{1}{2}}$ in the stencil. There are only two possibilities for adding another adjacent point: the stencil becomes either $\left\{x_{j-\frac{1}{2}}, x_{j+\frac{1}{2}}\right\}$ or $\left\{x_{j+\frac{1}{2}}, x_{j+\frac{3}{2}}\right\}$. The two corresponding first-order polynomials are given by $H\left(x_{j+\frac{1}{2}}\right)+H\left[x_{j-\frac{1}{2}}, x_{j+\frac{1}{2}}\right]\left(x-x_{j+\frac{1}{2}}\right)$ and $H\left(x_{j+\frac{1}{2}}\right)+$ $H\left[x_{j+\frac{1}{2}}, x_{j+\frac{3}{2}}\right]\left(x-x_{j+\frac{1}{2}}\right)$ respectively, where $H[*, *]$ are the Newton divided differences. Notice that these two polynomials differ only by the coefficients $H[*, *]$, which in this case are slopes and should be $O(1)$ if $f(u(x))$ is smooth but would be $O\left(\frac{1}{\Delta x}\right)$ if $f(u(x))$ contains a shock in the stencil. One is thus naturally led to take the one with a smaller $H[*, *]$ in absolute value. This idea can be easily pushed into high orders by induction, since when one has a $(k-1)$ th-order polynomial at hand and tries to extend it to a $k$ thorder one, one again only has to consider two possibilities of adding another adjacent point into the stencil. The two corresponding $k$ th-order polynomials differ only by the coefficients in the last term of the Newton form, which are the $k$ th-order Newton divided differences and are of the same magnitudes as the $k$ th derivatives for the smooth case but are of the order $O\left(1 / \Delta x^{k-m}\right)$ if the function is $C^{m-1}$ but not $C^{m}$ in the stencil. One thus again chooses the one with a smaller $k$ th divided difference in absolute value.

What can be proven about the ENO interpolation described above is that: for a piece-wise smooth function, the ENO approximation is uniformly accurate up to the discontinuities and is TVB (total variation bounded) even when the stencil must contain two points due to upwinding, hence will cross the discontinuity in the shocked cell itself.

Some upwinding is preferred for (2.1) in gaining stability. In the simplest situation: scalar one space dimension and $f^{\prime}(u) \geq 0$, one simply requires that the stencil of $P_{j+\frac{1}{2}}(x)$ contains $\left\{x_{j-\frac{1}{2}}, x_{j+\frac{1}{2}}\right\}$ in order to gain the necessary upwind biasing. The case with $f^{\prime}(u) \leq 0$ can be handled similarly. If $f^{\prime}(u)$ is not of one sign, one can do a flux splitting $f(u)=f^{+}(u)+f^{-}(u)$ where $f^{+\prime}(u) \geq 0$ and $f^{-\prime}(u) \leq 0$, and use upwinding on each of them separately. The simplest such splitting is due to Lax-Friedrichs: $f^{ \pm}(u)=$ $\frac{1}{2}(f(u) \pm \alpha u)$ where $\alpha=\max _{u}\left|f^{\prime}(u)\right|$, and this is the one we will use in this paper. Since for our case the conservation law (2.1) is a system, we will first find the eigenvalues and eigenvectors of the Jacobian $f^{\prime}(u)$. Using them we can perform the scalar procedure in the locally defined "characteristic fields". The details of this procedure can be found in [17] and [19].

The point-wise ENO scheme described above can be easily applied to multi space dimensions without additional difficulty. One simply approximates $f(u)_{x}$ along $x$ lines with all the other variables $(y, z$, etc.) fixed. The two- or three-dimensional computer 
programming is essentially only the one-dimensional code with one or two outside "do loops".

We still have to discretize the time variable $t$ in the ODE (2.2). We shall use the socalled TVD (total variation diminishing) Runge-Kutta type high-order time discretization introduced in [16]. This type of time discretization does not increase the total variation of the spatial operator. Hence if the spatial operator is TVD or TVB, so will the fully discretized scheme be, which is then high order in both space and time. In this paper we use third-order accurate schemes: for the ODE (2.2) rewritten in the form

$$
\frac{d u}{d t}=L(u)
$$

where $L(u)$ is the spatial operator, the third-order TVD Runge-Kutta method is simply

$$
\begin{aligned}
u^{(1)} & =u^{n}+\Delta t L\left(u^{n}\right), \\
u^{(2)} & =\frac{3}{4} u^{n}+\frac{1}{4} u^{(1)}+\frac{1}{4} \Delta t L\left(u^{(1)}\right), \\
u^{n+1} & =\frac{1}{3} u^{n}+\frac{2}{3} u^{(2)}+\frac{2}{3} \Delta t L\left(u^{(2)}\right) .
\end{aligned}
$$

The ENO schemes as described above may change stencils more than necessary in smooth regions near critical points, where the magnitudes of the divided differences are small. This may affect accuracy in some cases [18]. One remedy is to use a biasing factor when the stencil is chosen: when a point must be added to the stencil, with two candidate divided differences $a$ and $b$, if adding $a$ to the stencil results in a more central scheme, then $a$ is added unless, in absolute values, it is bigger than the factor times $b$ [18]. The factor can be any number between 1 and 2. For factor $=1$ it is the usual ENO scheme; for factor $\leq 2$ it will produce a TVD (total variation diminishing) scheme in the second-order case. Another further remedy is to apply a threshold: namely, if both divided differences $a$ and $b$ are less than this threshold, then add the one that makes the stencil more central. The details of these remedies can be found in [18] and [1].

Finally, we should mention how the memory terms in (1.1)-(1.3) are handled. For the special kernel $a$ of the form (1.5), which is the only case we consider in this paper, by defining

$$
z_{j}(x, t)=\int_{0}^{t} c_{j} \mu_{j} e^{-\mu_{j}(t-\tau)} \psi(u(x, \tau))_{x} d \tau,
$$

the equations (1.1)-(1.3) can be rewritten as

$$
\begin{gathered}
\left\{\begin{array}{c}
u_{t}-v_{x}=0, \\
v_{t}-\phi(u)_{x}=-\sum_{j=1}^{N} z_{j}+g, \quad x \in \mathcal{R}, t>0, \\
\left(z_{j}\right)_{t}-c_{j} \mu_{j} \psi(u)_{x}=-\mu_{j} z_{j}, \quad j=1, \ldots, N,
\end{array}\right. \\
u(x, 0)=u_{0}(x), \quad v(x, 0)=v_{0}(x), \quad z_{j}(x, 0)=0, \quad x \in \mathcal{R}, j=1, \ldots, N ;
\end{gathered}
$$

see, for example, [13]. This eliminates the memory terms from the system, at the price of $N$ additional ODE's. Computationally this form is much more convenient and economical. If the memory kernel $a$ is not of the special form (1.5), then this trick cannot 
be used and one must solve the original system (1.1)-(1.3), resulting in a much heavier storage requirement.

The left-hand side of the first two equations in (2.11) will be solved using the ENO schemes described above, with the local characteristic decompositions. The $\psi(u)_{x}$ term in the last $N$ equations in (2.11) will be the same as $\phi(u)_{x}$ in the second equation if $\psi=\phi$, or can be approximated by a scalar ENO interpolation procedure or even by central difference. Our computational experiments in Sec. 4 indicate that the result is not sensitive to either choice; the result shown is that obtained by using the fourth-order central difference in approximating $\psi(u)_{x}$ when $\psi \neq \phi$. Time discretization for all the equations is by the third-order TVD Runge-Kutta method (2.9).

3. Mathematical properties. In this section we study what we can expect for weak solutions to the initial-value problem (1.1)-(1.3). The linearized system is studied through its Green's function, and all the information about discontinuities in the solutions is obtained. The nonlinear system is also discussed.

Consider the following linearized system of (1.1), (1.3):

$$
\left\{\begin{aligned}
u_{t}-v_{x} & =0 \\
v_{t}-\phi^{\prime} u_{x} & =\int_{0}^{t} a^{\prime}(t-\tau) \psi^{\prime} u_{x}(x, \tau) d \tau,
\end{aligned}\right.
$$

where $\phi^{\prime}>0, \psi^{\prime}>0$ are constants, $a$ is a kernel of the form (1.5), and

$$
p^{\prime} \equiv \phi^{\prime}-a(0) \psi^{\prime}>0 \text {. }
$$

To study the solution of the Cauchy problem of (3.1), all we need is to understand the Green's function of (3.1), by which we mean the solution matrix $G(x, t)$ to $(3.1)$ satisfying the initial data

$$
G(x, 0)=\delta(x) I
$$

where $\delta$ is the Dirac $\delta$-function and $I$ is the $2 \times 2$ identity matrix. The following is the main theorem of this section.

Theorem 3.1. Assume that $\phi^{\prime}>0, \psi^{\prime}>0$, (3.2) holds, and the kernel $a$ is given by (1.5). For all $-\infty<x<\infty, t \geq 0$, the Green's function $G(x, t)$ of (3.1) has the property

$$
\begin{aligned}
G(x, t)=\frac{1}{\sqrt{\mu t}} & \left\{e^{-\frac{\left(x+t \sqrt{p^{\prime}}\right)^{2}}{2 \mu t}} P_{-, p^{\prime}}+e^{-\frac{\left(x-t \sqrt{p^{\prime}}\right)^{2}}{2 \mu t}} P_{+, p^{\prime}}\right\} \\
+ & O(1)(t+1)^{-\frac{1}{2}} t^{-\frac{1}{2}}\left\{e^{-\frac{\left(x+t{\sqrt{p^{\prime}}}^{2}\right.}{C t}}+e^{-\frac{\left(x-t \sqrt{p^{\prime}}\right)^{2}}{C t}}\right\} \\
& +e^{\frac{\psi^{\prime} a^{\prime}(0) t}{2 \phi^{\prime}}}\left\{\delta\left(x+t \sqrt{\phi^{\prime}}\right) P_{-, \phi^{\prime}}+\delta\left(x-t \sqrt{\phi^{\prime}}\right) P_{+, \phi^{\prime}}\right\},
\end{aligned}
$$

where $p^{\prime}$ is defined in (3.2), $C>0$ is a constant independent of $x$ and $t$,

$$
\mu=\psi^{\prime} \int_{0}^{\infty} a(s) d s=\psi^{\prime} \sum_{j=1}^{N} c_{j} / \mu_{j}
$$


and

$$
P_{\mp, c} \equiv\left(\begin{array}{cc}
\frac{1}{2} & \pm \frac{1}{2 \sqrt{c}} \\
\pm \frac{\sqrt{c}}{2} & \frac{1}{2}
\end{array}\right) \quad \text { for } c>0 .
$$

The proof of this theorem will be outlined at the end of this section. We now discuss what we can learn from (3.3). The solution of the Cauchy problem of (3.1) is the convolution with respect to $x$ of $G(x, t)$ with the initial data. It is clear that the largetime behavior of the solution is governed by the leading term of $G$, the first two heat kernels in (3.3). Here notice that $a^{\prime}(0)<0$ and the last term decays exponentially. To have a heuristic understanding of the large-time behavior, we use asymptotic expansions to obtain a viscous approximation of the system (1.1), (1.3). Using integration by parts, (1.1), (1.3) can be written as

$$
\left\{\begin{aligned}
u_{t}-v_{x} & =0 \\
v_{t}-\phi(u)_{x} & =-a(0) \psi(u)_{x}+a(t) \psi(u(x, 0))_{x}+\int_{0}^{t} a(t-\tau) \psi(u(x, \tau))_{x \tau} d \tau .
\end{aligned}\right.
$$

Here we have assumed $g=0$. Since $a$ decays exponentially in $t$, it is reasonable to replace $\psi(u(x, \tau))$ in the above integral by its Taylor approximation around $t$,

$$
\psi(u(x, t))+(\tau-t) \psi(u(x, t))_{t}
$$

when $t$ is large. Using the first equation, we then arrive at an approximate system of (1.1), (1.3):

$$
\left\{\begin{aligned}
u_{t}-v_{x} & =0 \\
v_{t}-p(u)_{x} & =\left(\mu(u) v_{x}\right)_{x}
\end{aligned}\right.
$$

where

$$
p(u) \equiv \phi(u)-a(0) \psi(u), \quad \mu(u) \equiv \psi^{\prime}(u) \int_{0}^{\infty} a(s) d s .
$$

System (3.6), (3.7) was proposed by Liu [10] without justification. If the initial data are smooth and small, Kawashima [7] showed that the solution to (3.6) has the same large-time behavior in $L^{2}$ as the solution to

$$
\left\{\begin{aligned}
u_{t}-v_{x} & =\frac{\mu(0)}{2} u_{x x} \\
v_{t}-p(u)_{x} & =\frac{\mu(0)}{2} v_{x x}
\end{aligned}\right.
$$

with the same initial data. Zeng [22] further showed that this is true in $L^{p}, 1 \leq p \leq \infty$, and in fact, the leading term of the Green's function of the linearization of (3.6) is the Green's function of the linearization of (3.8). Therefore, it is not surprising that in (3.3) the leading term of the Green's function of (3.1) is exactly the Green's function of the linearization of (3.8). Estimate (3.3) is a powerful tool to study the large-time behavior of solutions to the nonlinear problem (1.1)-(1.3) when the initial data are smooth and small. In this case it has been shown that the problems (1.1)-(1.3) and (3.8), (1.2) have the same asymptotic solution in $L^{2}$, i.e., the sum of the two diffusion waves associated with (3.8) which are solutions to the Burgers' equation; see [21]. With (3.3), we can obtain much better results. We can show that the difference between the solution of (1.1)-(1.3) and the diffusion waves has a point-wise estimate both in $x$ and $t$, which 
is a sum of diffusion waves of algebraic types decaying more rapidly than the solution. Based on this estimate, not only all the $L^{p}(1 \leq p \leq \infty)$ decay rates of the difference are straightforward and optimal, we can also see different decay rates in different directions in the $(x, t)$-plane. The exact formulation and proof of this estimate are totally parallel to those for hyperbolic-parabolic systems given in a recent paper by Liu and Zeng [11] (also see [23]), and will not be discussed here.

Since local behavior of the solution is concerned, we see that in the linear case the two $\delta$-functions in (3.3) describe how an initial discontinuity propagates into the solution since all the other terms smooth away immediately any initial discontinuity. These two $\delta$-functions are a multiple by a factor $e^{\psi^{\prime} a^{\prime}(0) t /\left(2 \phi^{\prime}\right)}$ of the Green's function of the linearization of

$$
\left\{\begin{aligned}
u_{t}-v_{x} & =0 \\
v_{t}-\phi(u)_{x} & =0
\end{aligned}\right.
$$

Notice that $a^{\prime}(0)$ is negative. Thus any initial discontinuity splits into two parts, propagating in the directions $d x / d t=\mp \sqrt{\phi^{\prime}}$ respectively and decaying exponentially in time. In Sec. 4 , we have a numerical example for a linear problem with initial discontinuities. Formula (3.3) then predicts the locations and amplitudes of the discontinuities in the solution and how they interact. The numerical result matches the theoretical result so well that it shows the robustness of our numerical scheme for singularities. Because of the decay of the amplitude, a discontinuity in the linear case is more or less of local behavior. Indeed, system (3.9) is the elastic model with the instantaneous elastic response in (1.3).

For nonlinear systems, certain conditions have to be satisfied when crossing a discontinuity (shock wave), since (1.1) is a system of conservation laws. Here again we assume $g=0$. It has been shown that the memory term has nothing to do with these conditions. More precisely, if a solution $(u, v)$ of $(1.1),(1.3)$ is discontinuous along a curve $x=x(t)$, then it satisfies the following jump condition:

$$
\begin{gathered}
x^{\prime}(t)\left(u_{r}-u_{l}\right)=-\left(v_{r}-v_{l}\right) \\
x^{\prime}(t)\left(v_{r}-v_{l}\right)=-\left(\phi\left(u_{r}\right)-\phi\left(u_{l}\right)\right) \\
\left(u_{r}, v_{r}\right) \equiv(u, v)(x(t)+0, t), \quad\left(u_{l}, v_{l}\right) \equiv(u, v)(x(t)-0, t) ;
\end{gathered}
$$

see [10]. Condition (3.10) is exactly the same jump condition for the system (3.9), which governs the local behavior. With the estimate (3.3), we further expect that any jump generated by a small initial discontinuity decays exponentially in $t$.

We now consider an interesting problem, the Riemann problem (1.1)-(1.3) with the initial data

$$
u_{0}(x)=\left\{\begin{array}{ll}
u_{-}, & x<0, \\
u_{+}, & x>0,
\end{array}, \quad v_{0}(x)= \begin{cases}v_{-}, & x<0 \\
v_{+}, & x>0\end{cases}\right.
$$


We assume that the constant states $\left(u_{-}, v_{-}\right)$and $\left(u_{+}, v_{+}\right)$consist of a 1-shock of the inviscid system corresponding to (3.6):

$$
\left\{\begin{aligned}
u_{t}-v_{x} & =0 \\
v_{t}-p(u)_{x} & =0
\end{aligned}\right.
$$

where $p$ is defined in (3.7). That is, the shock speed $c<0$ and the Riemann data (3.11) satisfy the Rankine-Hugoniot condition and admissibility criterion:

$$
\begin{gathered}
c\left(u_{+}-u_{-}\right)=-\left(v_{+}-v_{-}\right), \quad c\left(v_{+}-v_{-}\right)=-\left(p\left(u_{+}\right)-p\left(u_{-}\right)\right), \\
c^{2} \equiv \frac{p\left(u_{+}\right)-p\left(u_{-}\right)}{u_{+}-u_{-}} \geq \frac{p(u)-p\left(u_{-}\right)}{u-u_{-}} \quad \text { for all } u \text { between } u_{-} \text {and } u_{+} .
\end{gathered}
$$

Liu [10] has shown that if in addition to condition (3.13), the following nonresonance condition

$$
c^{2}<\phi^{\prime}(u) \quad \text { for all } u \text { between } u_{-} \text {and } u_{+} \text {and } u=u_{ \pm}
$$

is satisfied, e.g., when $\left(u_{+}, v_{+}\right)$is close to $\left(u_{-}, v_{-}\right)$, then system (1.1), (1.3) has a smooth traveling wave solution

$$
(u, v)(x, t)=\left(\theta_{1}, \theta_{2}\right)(x-c t), \quad\left(\theta_{1}, \theta_{2}\right)( \pm \infty)=\left(u_{ \pm}, v_{ \pm}\right),
$$

which is strictly monotone and unique up to a translation; if (3.13) holds but (3.14) fails, then the system (1.1), (1.3) has a traveling wave solution (3.15) which satisfies the following conditions across any discontinuity $\left(u_{l}, v_{l} ; u_{r}, v_{r}\right)$ contained therein:

$$
\begin{gathered}
c\left(u_{r}-u_{l}\right)=-\left(v_{r}-v_{l}\right), \quad c\left(v_{r}-v_{l}\right)=-\left(\phi\left(u_{r}\right)-\phi\left(u_{l}\right)\right), \\
\frac{\phi\left(u_{r}\right)-\phi\left(u_{l}\right)}{u_{r}-u_{l}} \geq \frac{\phi(u)-\phi\left(u_{l}\right)}{u-u_{l}}, \quad \text { for all } u \text { between } u_{l} \text { and } u_{r},
\end{gathered}
$$

and such a solution is monotone and unique up to translation. When we consider the Riemann problem of (1.1), (1.3), the initial data (3.11) can be viewed as a perturbation of the profile $\left(\theta_{1}, \theta_{2}\right)$ in (3.15). It is a conjecture that the solution to the Riemann problem approaches the aforementioned traveling wave as $t \rightarrow \infty$, where the translation is uniquely determined by the mass of the initial perturbation (private communication with T.-P. Liu). In Examples 4 and 5 of Sec. 4, we solve Riemann problems numerically with initial data satisfying condition (3.13). The nonresonance condition (3.14) is satisfied by Example 4, but not by Example 5 . We do see different large-time behavior there; see these two examples for details.

In the rest of this section we outline the proof of Theorem 3.1. The approach is similar to that for hyperbolic-parabolic systems (see [11]): We first need a good understanding of the Fourier transform of $G$, and then estimate the inverse transform. The Fourier transform of $G$ has been studied by Zeng, [21]. Here we cite relevant results from [21] and refine some of the estimates for our purpose here. Once we have the desired estimates of the Fourier transform, the estimates of the inverse transform are obtained in the same way as for hyperbolic-parabolic systems, and the readers are referred to [11].

Following the notation in [21], we write the Laplace transform of $a$ of the form (1.5) as

$$
L_{a}(s)=q_{1}(s) / q_{2}(s)
$$


where $q_{1}$ and $q_{2}$ are polynomials relatively prime and of degrees $m-1$ and $m$ respectively. For definiteness we assume that in $q_{2}$ the coefficient of $s^{m}$ is 1 . Set

$$
d(s ; \xi)=s^{2} q_{2}(s)+\psi^{\prime} \xi^{2} s q_{1}(s)+p^{\prime} \xi^{2} q_{2}(s) .
$$

Then $d$ is a polynomial of $s$ with degree $n=m+2$. From Lemma 2.1 in [21], $d$ has exactly $n$ different zeros $\lambda_{k}(\xi), k=1, \ldots, n$, except for a finite number of $\xi$. From Lemma 2.2 in [21], we have the following expression for the Fourier transform of the Green's function:

LEMMA 3.2. Under the hypotheses of Theorem 3.1, the Fourier transform $\widehat{G}(\xi, t)$ of $G(x, t)$ with respect to $x$ has the expression

$$
\widehat{G}(\xi, t)=\sum_{k=1}^{n} e^{\lambda_{k}(\xi) t} P_{k}(\xi)
$$

where the $\lambda_{k}(\xi)$ are the zeros of $d(s ; \xi)$ defined in (3.19),

$$
P_{k}(\xi)=\frac{1}{d^{\prime}\left(\lambda_{k} ; \xi\right)}\left(\begin{array}{cc}
\lambda_{k} q_{2}\left(\lambda_{k}\right) & i \xi q_{2}\left(\lambda_{k}\right) \\
i \xi\left(\psi^{\prime} \lambda_{k} q_{1}\left(\lambda_{k}\right)+p^{\prime} q_{2}\left(\lambda_{k}\right)\right) & \lambda_{k} q_{2}\left(\lambda_{k}\right)
\end{array}\right)
$$

$d^{\prime}$ is the derivative of $d$ with respect to $s$, and $q_{1}, q_{2}$ are as in (3.18).

LEMma 3.3. $\widehat{G}(\xi, t)$ in Lemma 3.2 is an entire function of $\xi$.

Proof. By definition, $\lambda_{k}(\xi), k=1, \ldots, n$, are the zeros of $d(s ; \xi)$. Clearly, the $\lambda_{k}(\xi)$ are continuous algebraic functions in the $\xi$-plane since the coefficient of $s^{n}$ in $d$ is 1 . The $\lambda_{k}(\xi)$ are all distinct if $\xi$ is not one of the exceptional points, where some of the $\lambda_{k}$ coincide. As mentioned above, there are only a finite number of exceptional points in the whole $\xi$-plane. An exceptional point is either an algebraic singularity of some of the $\lambda_{k}$, or a regular point of all of them. In each simply connected domain containing no exceptional points, the $\lambda_{k}(\xi)$ are holomorphic; so is $\widehat{G}(\xi, t)$. Notice that when $\xi$ describes a small circle around an exceptional point, the $\lambda_{k}(\xi)$ undergo a permutation among themselves after analytic continuation. By $(3.20)$ and $(3.21), \widehat{G}(\xi, t)$ is single valued. We can further show that $\widehat{G}(\xi, t)$ is bounded in a small neighborhood of an exceptional point, and hence analytic there; see the proof of Lemma 2.4 in [21].

Q.E.D.

The behavior of the $\lambda_{k}(\xi)$ and $P_{k}(\xi)$ in (3.20) when $\xi$ is small determines the leading term of $G(x, t)$, while the behavior when $\xi$ is large determines the $\delta$-functions in $G(x, t)$. The following lemma is Lemma 2.4 and part of Lemma 2.3 in [21].

LEMMA 3.4. Under the hypotheses of Theorem 3.1, we have the following properties:

(i) As functions of the complex variable $\xi, \lambda_{1,2}(\xi)$ and $P_{1,2}(\xi)$ are analytic at the origin and have the Taylor expansions

$$
\begin{aligned}
& \lambda_{1,2}(\xi)= \pm i \sqrt{p^{\prime}} \xi-\frac{1}{2} \mu \xi^{2}+O\left(\xi^{3}\right) \\
& P_{1,2}(\xi)=\left(\begin{array}{cc}
\frac{1}{2} & \pm \frac{1}{2 \sqrt{p^{\prime}}} \\
\pm \frac{\sqrt{p^{\prime}}}{2} & \frac{1}{2}
\end{array}\right)+O(\xi)
\end{aligned}
$$

for small $\xi$. 
(ii) Let $\xi$ be real and $\varepsilon>0$ be small. Then there exists a constant $C=C(\varepsilon)$, such that

$$
\begin{aligned}
\left|\sum_{k=3}^{n} P_{k}(\xi) e^{\lambda_{k}(\xi) t}\right| \leq C e^{-t / C}, \quad \text { for }|\xi| \leq \varepsilon \text { and } t \geq 0 \\
|\widehat{G}(\xi, t)| \leq C e^{-t / C}, \quad \text { for }|\xi| \geq \varepsilon \text { and } t \geq 0 .
\end{aligned}
$$

LEMmA 3.5. Under the hypotheses of Theorem 3.1, as the complex variable $\xi \rightarrow \infty$, we have

$$
\begin{gathered}
\lambda_{1,2}(\xi)= \pm i \sqrt{\phi^{\prime}} \xi+\frac{\psi^{\prime} a^{\prime}(0)}{2 \phi^{\prime}}+O\left(\xi^{-1}\right), \\
\lambda_{k}(\xi)=\lambda_{k}^{0}+O\left(\xi^{-\gamma}\right), \quad k=3, \ldots, n, \\
P_{1,2}(\xi)=\left(\begin{array}{cc}
\frac{1}{2} & \pm \frac{1}{2 \sqrt{\phi^{\prime}}} \\
\pm \frac{\sqrt{\phi^{\prime}}}{2} & \frac{1}{2}
\end{array}\right)+\xi^{-1} C_{M}+O\left(\xi^{-2}\right), \\
\sum_{k} e^{\lambda_{k}(\xi) t} P_{k}(\xi)=e^{\lambda_{k}^{0} t} \xi^{-1} C_{M}+O\left(\xi^{-1-\gamma}\right) e^{\frac{1}{2}\left(\operatorname{Re} \lambda_{k}^{0}\right) t},
\end{gathered}
$$

where $0<\gamma \leq 1$ is a constant, the $\lambda_{k}^{0}$ are the $m$ zeros of

$$
q(s) \equiv p^{\prime} q_{2}(s)+\psi^{\prime} s q_{1}(s),
$$

the $\lambda_{k}^{0}$ have negative real parts, $C_{M}$ denotes a universal polynomial matrix in $t$ with degree not more than $n-3$, and the summation in (3.28) is for all $3 \leq k \leq n$ such that $\lambda_{k}(\xi)$ in (3.26) corresponds to the same value of $\lambda_{k}^{0}$.

Proof. This lemma is a refinement of Lemma 2.3 in [21], where it has been shown that $\lambda_{1,2}(\xi) / \xi \rightarrow \pm i \sqrt{\phi^{\prime}}$ and $\lambda_{k}(\xi) \rightarrow \lambda_{k}^{0}, k=3, \ldots, n$, as $\xi \rightarrow \infty$, and the $\lambda_{k}^{0}$ have negative real parts. Notice that $\lambda_{k}$ has algebraic singularities. If one of them is infinity, then in (3.26) we have $0<\gamma<1$; otherwise $\gamma=1$. To prove (3.25), notice that $\lambda_{1,2}$ are zeros of d. From (3.19),

$$
\frac{1}{\xi^{2}}=-\frac{\psi^{\prime} \lambda_{1,2} q_{1}\left(\lambda_{1,2}\right)+p^{\prime} q_{2}\left(\lambda_{1,2}\right)}{\lambda_{1,2}^{2} q_{2}\left(\lambda_{1,2}\right)} .
$$

Clearly, $1 / \xi$ is an analytic function of $1 / \lambda_{1,2}$ as $\lambda_{1,2} \rightarrow \infty(\xi \rightarrow \infty)$. The inverse function theorem then implies that $1 / \lambda_{1,2}$ are analytic functions of $1 / \xi$. Hence $\lambda_{1,2}$ are singlevalued at infinity:

$$
\lambda_{1,2}= \pm i \sqrt{\phi^{\prime}} \xi+c_{1,2}+O\left(\xi^{-1}\right),
$$

where $c_{1,2}$ are constants. To determine $c_{1,2}$, set

$$
\tilde{\lambda}_{1,2} \equiv \lambda_{1,2} \mp i \sqrt{\phi^{\prime}} \xi \text {. }
$$

Then $c_{1,2}=\lim _{\xi \rightarrow \infty} \tilde{\lambda}_{1,2}$. From (3.30) and (3.18), we have

$$
\left( \pm i \sqrt{\phi^{\prime}} \xi+\tilde{\lambda}_{1,2}\right)^{2}=-\xi^{2}\left(\psi^{\prime} \lambda_{1,2} L_{a}\left(\lambda_{1,2}\right)+p^{\prime}\right) .
$$

By (3.2) and properties of the Laplace transform, we have

$$
\pm 2 i \sqrt{\phi^{\prime}} \xi \tilde{\lambda}_{1,2}+\tilde{\lambda}_{1,2}^{2}=-\xi^{2}\left(\psi^{\prime} \lambda_{1,2} L_{a}\left(\lambda_{1,2}\right)-a(0) \psi^{\prime}\right)=-\xi^{2} \psi^{\prime} L_{a^{\prime}}\left(\lambda_{1,2}\right) .
$$


Notice that $\lim _{s \rightarrow \infty} s L_{a^{\prime}}(s)=a^{\prime}(0)$. We arrive at

$$
c_{1,2}=\lim _{\xi \rightarrow \infty} \mp \frac{\psi^{\prime} \xi}{2 i \sqrt{\phi^{\prime}}} L_{a^{\prime}}\left(\lambda_{1,2}\right)=\frac{\psi^{\prime} a^{\prime}(0)}{2 \phi^{\prime}} .
$$

Together with (3.31), estimate (3.25) is proved. The estimate $(3.27)$ for $P_{1,2}(\xi)$ is straightforward by the estimates for $\lambda_{k}, k=1, \ldots, n$, and (3.21), where we notice that $d^{\prime}\left(\lambda_{1,2} ; \xi\right)$ are single-valued at infinity. To prove (3.28), we assume for definiteness that $\lambda^{0} \equiv \lambda_{3}^{0}=\cdots=\lambda_{l}^{0}$, and $\lambda_{k}^{0} \neq \lambda^{0}$ for $l+1 \leq k \leq n$. By (3.25) and (3.26), we have the following for large $\xi$ :

$$
\begin{aligned}
\left|\lambda_{1,2}(\xi)\right| & >\frac{\sqrt{\phi^{\prime}}}{2}|\xi|, \\
\left|\lambda_{k}(\xi)-\lambda^{0}\right| & <\varepsilon, \quad k=3, \ldots, l, \\
\left|\lambda_{k}(\xi)-\lambda^{0}\right| & >2 \varepsilon, \quad k=l+1, \ldots, n,
\end{aligned}
$$

where $\varepsilon>0$ is a fixed small number. Then by (3.21) and the residue theorem, the element in the first row and second column of the left-hand side of $(3.28)$ is

$$
\sum_{k=3}^{l} \frac{i \xi q_{2}\left(\lambda_{k}\right) e^{\lambda_{k} t}}{d^{\prime}\left(\lambda_{k} ; \xi\right)}=\frac{1}{2 \pi i} \oint_{\left|\lambda-\lambda^{0}\right|=3 \varepsilon / 2} \frac{i \xi q_{2}(\lambda) e^{\lambda t}}{d(\lambda ; \xi)} d \lambda=\mathrm{I}+\mathrm{II}
$$

where

$$
\begin{gathered}
\mathrm{I}=\frac{1}{2 \pi i} \oint_{\left|\lambda-\lambda^{0}\right|=3 \varepsilon / 2} \frac{q_{2}(\lambda) e^{\lambda t}}{-i \xi \phi^{\prime}\left(\lambda-\lambda^{0}\right)^{l-2}\left(\lambda-\lambda_{l+1}^{0}\right) \cdots\left(\lambda-\lambda_{n}^{0}\right)} d \lambda, \\
\mathrm{II}=\frac{1}{2 \pi i} \oint_{\left|\lambda-\lambda^{0}\right|=3 \varepsilon / 2} \frac{q_{2}(\lambda) e^{\lambda t}}{i \xi}\left[\frac{(i \xi)^{2}}{\left(\lambda-\lambda_{1}(\xi)\right) \cdots\left(\lambda-\lambda_{n}(\xi)\right)}\right. \\
\left.+\frac{1}{\phi^{\prime}\left(\lambda-\lambda^{0}\right)^{l-2}\left(\lambda-\lambda_{l+1}^{0}\right) \cdots\left(\lambda-\lambda_{n}^{0}\right)}\right] d \lambda .
\end{gathered}
$$

By Cauchy's formula,

$$
\mathrm{I}=-\frac{1}{i \xi \phi^{\prime}(l-3) !} \frac{d^{l-3}}{d \lambda^{l-3}}\left[\frac{q_{2}(\lambda) e^{\lambda t}}{\left(\lambda-\lambda_{l+1}^{0}\right) \cdots\left(\lambda-\lambda_{n}^{0}\right)}\right]_{\lambda=\lambda^{0}}=c \xi^{-1} e^{\lambda^{0} t}
$$

where $c$ is a polynomial in $t$ with degree not more than $l-3$. To estimate II, we use (3.25), (3.26), and (3.32) to obtain

$$
|\mathrm{II}| \leq \frac{3 \varepsilon}{2}|\xi|^{-1} \max _{\left|\lambda-\lambda^{0}\right|=3 \varepsilon / 2}\left|q_{2}(\lambda) e^{\lambda t}\right| O\left(\xi^{-\gamma}\right) \varepsilon^{-n+1}=O(1)|\xi|^{-1-\gamma} e^{\frac{1}{2}\left(\operatorname{Re} \lambda^{0}\right) t}
$$

for large $\xi$. Equations (3.33)-(3.35) and similar treatment for the other elements give us (3.28). 
With Lemmas 3.2-3.5, the proof of (3.3) is totally parallel to the case of hyperbolicparabolic systems: Lemmas $3.2,3.4$, and 3.5 give us

$$
\begin{array}{r}
R \equiv G(x, t)-\frac{1}{\sqrt{\mu t}}\left\{e^{-\frac{\left(x+t \sqrt{p^{\prime}}\right)^{2}}{2 \mu t}} P_{-, p^{\prime}}+e^{-\frac{\left(x-t \sqrt{p^{\prime}}\right)^{2}}{2 \mu t}} P_{+, p^{\prime}}\right\} \\
-e^{\frac{\psi^{\prime} a^{\prime}(0) t}{2 \phi^{\prime}}}\left\{\delta\left(x+t \sqrt{\phi^{\prime}}\right) P_{-, \phi}+\delta\left(x-t \sqrt{\phi^{\prime}}\right) P_{+, \phi^{\prime}}\right\} \\
=O(1)(t+1)^{-1}\left\{e^{-\frac{\left(x+t \sqrt{p^{\prime}}\right)^{2}}{C t}}+e^{-\frac{\left(x-t \sqrt{p^{\prime}}\right)^{2}}{C t}}\right\} \\
+O(1)\left(t^{-\frac{1}{2}}+\left|x+t \sqrt{\phi^{\prime}}\right|+\left|x-t \sqrt{\phi^{\prime}}\right|+|x|\right) e^{-t / C}
\end{array}
$$

for all $-\infty<x<\infty, t>0$, where $C>0$ is a constant; see the proof of Lemma 5.5 in [11]. Lemmas 3.3 and 3.5 give us

$$
R=O(1) t^{-\frac{1}{2}}\left\{e^{-\frac{x^{2}}{C t}}+e^{-\frac{\left(x+t \sqrt{p^{\prime}}\right)^{2}}{C t}}+e^{-\frac{\left(x-t \sqrt{p^{\prime}}\right)^{2}}{C t}}\right\}
$$

for $-\infty<x<\infty, t>0$ and $x / t$ large; see the proof of Lemma 5.6 in [11]. The estimates (3.36) and (3.37) then imply (3.3); see the proof of Theorem 5.7 in [11].

4. Numerical results. In this section we present numerical results obtained by the third-order ENO scheme using the Lax-Friedrichs flux splitting. The CFL number (the upper bound of $\max _{j}\left|\lambda_{j}\right| \frac{\Delta t}{\Delta x}$ where $\lambda_{j}$ are the eigenvalues of the Jacobian $f^{\prime}(u)$ in (2.1)) is taken as 0.5.

EXAMPLE 1. This example is an accuracy test. We solve the initial-value problem (1.1)-(1.3), where

$$
\phi(u)=\psi(u)=u+\frac{1}{3} u^{3}, \quad a(t)=0.4 e^{-t} .
$$

The source term $g(x, t)$ and the initial data $u_{0}(x), v_{0}(x)$ are chosen such that the problem has the exact solution

$$
u(x, t)=e^{-t} \sin x, \quad v(x, t)=e^{-t} \cos x .
$$

We compute the solution on the interval $[-\pi, \pi]$ up to $t=1$, imposing periodic boundary conditions. The biasing factor is taken as 2 , and the threshold $\varepsilon$ is chosen to be small and decaying with the mesh size. Table 1 shows the errors for $u$ and $v$, respectively, in the discrete $L^{2}$ norm when $t=1$, with the corresponding total number $N$ of mesh points and the threshold $\varepsilon$. The order of accuracy is evaluated by $\log (e(\Delta x) / e(0.5 \Delta x)) / \log 2$.

TABLE 1. The accuracy for the smooth problem

\begin{tabular}{||c||c||c|c||c|c|}
\hline \hline$N$ & $\varepsilon$ & $L^{2}$ error for $u$ & Order & $L^{2}$ error for $v$ & order \\
\hline 80 & $10^{-4}$ & $6.872 \times 10^{-6}$ & & $1.095 \times 10^{-5}$ & \\
\hline 160 & $10^{-5}$ & $6.589 \times 10^{-7}$ & 3.38 & $1.110 \times 10^{-6}$ & 3.30 \\
\hline 320 & $10^{-6}$ & $7.913 \times 10^{-8}$ & 3.06 & $1.264 \times 10^{-7}$ & 3.13 \\
\hline \hline
\end{tabular}

Here we do see third-order convergence. We have also tested the problem with a different set of $g(x, t), u_{0}(x), v_{0}(x)$ such that the exact solution is non-periodic. Again we obtain third-order accuracy. This example indicates that for smooth solutions the scheme has the expected high-order accuracy. 
EXAMPLE 2. In this example we test the resolution of discontinuities by the scheme. We solve the linear problem (1.1)-(1.3) with

$$
\phi(u)=\psi(u)=4 u, \quad a(t)=0.4 e^{-t}+0.3 e^{-2 t}, \quad g(x, t)=0,
$$

and a set of periodic initial data with period 2 :

$$
u_{0}(x)=\left\{\begin{array}{ll}
-1, & -1<x<0, \\
1, & 0<x<1,
\end{array} \quad v_{0}(x)=0 .\right.
$$

The exact solution can be written as

$$
\left(\begin{array}{l}
u \\
v
\end{array}\right)(x, t)=\int_{-\infty}^{\infty} G(x-y, t)\left(\begin{array}{l}
u_{0} \\
v_{0}
\end{array}\right)(y) d y,
$$

where $G(x, t)$ is the Green's function of the system. Applying Theorem 2.1 to this problem, we have

$$
\begin{aligned}
& G(x, t)=\text { heat kernels }+ \text { higher-order terms of heat kernels } \\
& \qquad+e^{-t / 2}\left\{\delta(x+2 t)\left(\begin{array}{cc}
\frac{1}{2} & \frac{1}{4} \\
1 & \frac{1}{2}
\end{array}\right)+\delta(x-2 t)\left(\begin{array}{cc}
\frac{1}{2} & -\frac{1}{4} \\
-1 & \frac{1}{2}
\end{array}\right)\right\} .
\end{aligned}
$$

It is clear that the first two terms in the above expression do not contribute to any discontinuity, while the third term gives us all the information about the discontinuities in the solution. More precisely, discontinuities propagate along the straight lines $x=\mp 2 t+k$ for any integer $k$. The jumps along these lines are:

$$
\begin{aligned}
& {[u] \equiv u_{r}-u_{l}= \begin{cases}e^{-t / 2} & \text { along } x=\mp 2 t, \\
-e^{-t / 2} & \text { along } x=\mp 2 t \pm 1,\end{cases} } \\
& {[v] \equiv v_{r}-v_{l}= \begin{cases}2 e^{-t / 2} & \text { along } x=2 t-1 \text { and } x=-2 t \\
-2 e^{-t / 2} & \text { along } x=2 t \text { and } x=-2 t+1\end{cases} }
\end{aligned}
$$

for one period. Notice that the first interaction time of discontinuities is $\frac{1}{4}$. When $t=\frac{1}{4}, u$ is continuous, while $v$ has jumps at $x=\mp \frac{1}{2}$. The second interaction time is $\frac{1}{2}$. When $t=\frac{1}{2}, u$ has jumps at $x= \pm 1,0$; while $v$ is continuous everywhere. In this example, it is clear that the solution takes the constant state $(-1,0)$ in the region $\left\{2 t-1<x<-2 t, 0 \leq t<\frac{1}{4}\right\}$, and takes the constant state $(1,0)$ in the region $\left\{2 t<x<-2 t+1,0 \leq t<\frac{1}{4}\right\}$; see Figure 1 on p. 474. Therefore, we can further find out $u_{r}, u_{l}, v_{r}, v_{l}$ along the discontinuities for $t \leq \frac{1}{4}$. Especially,

$$
\begin{aligned}
& u\left(-\frac{1}{2}, \frac{1}{4}\right)=e^{-1 / 8}-1, \quad u\left(\frac{1}{2}, \frac{1}{4}\right)=1-e^{-1 / 8}, \\
& v_{r}\left(-\frac{1}{2}, \frac{1}{4}\right)=v_{l}\left(\frac{1}{2}, \frac{1}{4}\right)=2 e^{-1 / 8} \\
& v_{l}\left(-\frac{1}{2}, \frac{1}{4}\right)=v_{r}\left(\frac{1}{2}, \frac{1}{4}\right)=-2 e^{-1 / 8} .
\end{aligned}
$$

We compute the numerical solution for this example on the interval $[-1,1]$ up to $t=\frac{1}{2}$. A periodic boundary condition is imposed. We use zero threshold and take the factor as 1 , since these tend to be more robust for long-time integration with shocks. Numerical 


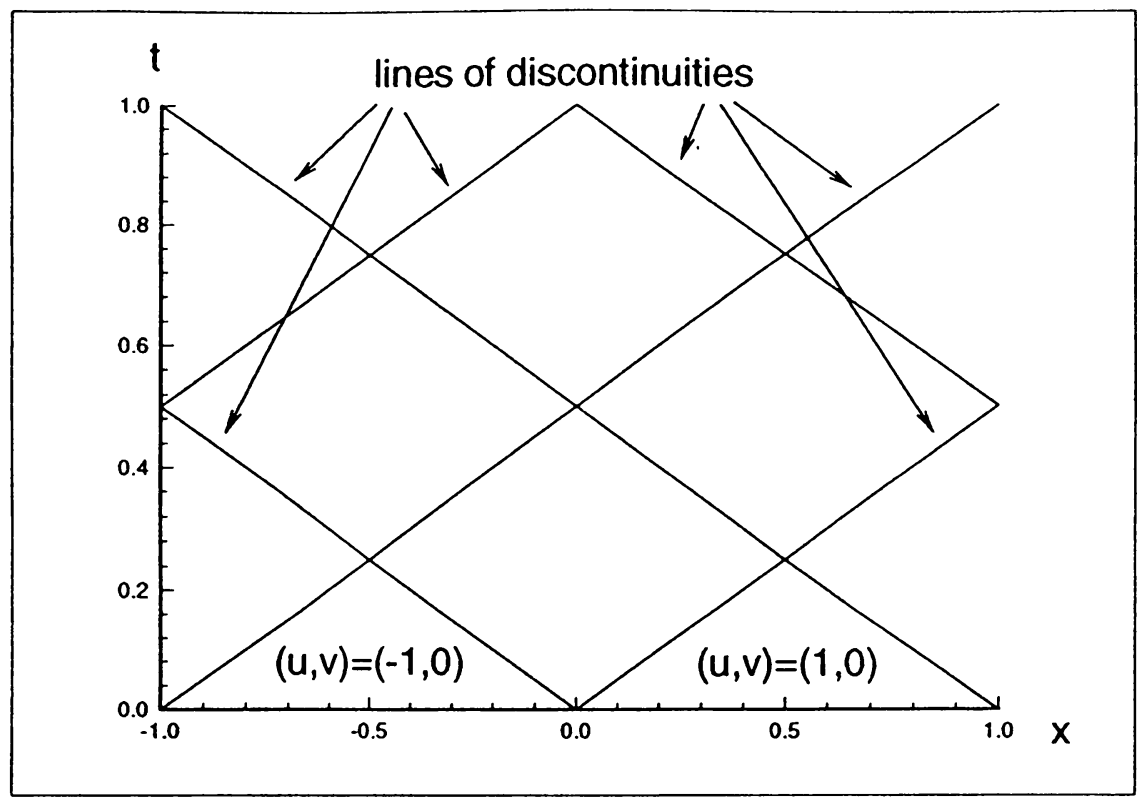

FIG. 1. The pattern of discontinuities and constant regions for the linear problem in Example 4.2.

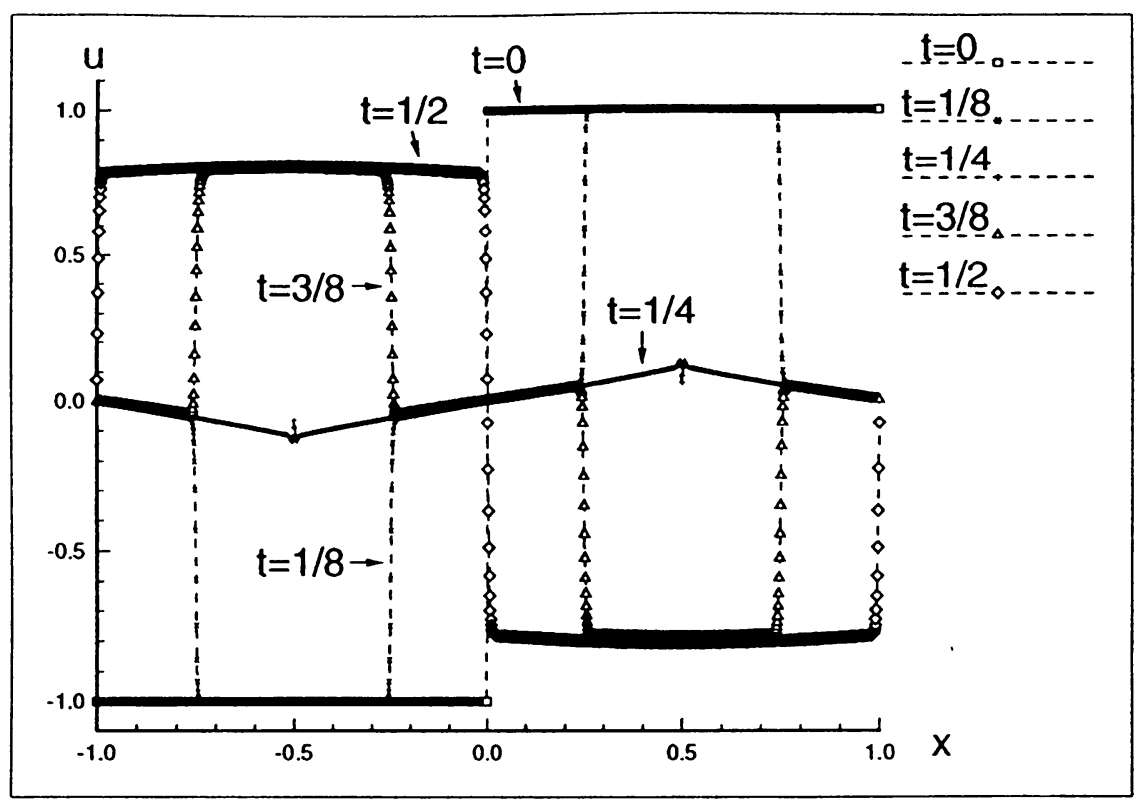

FIG. 2. The solution $u$ of Example 2, at $t=0, \frac{1}{8}, \frac{1}{4}, \frac{3}{8}$, and $\frac{1}{2}$. 


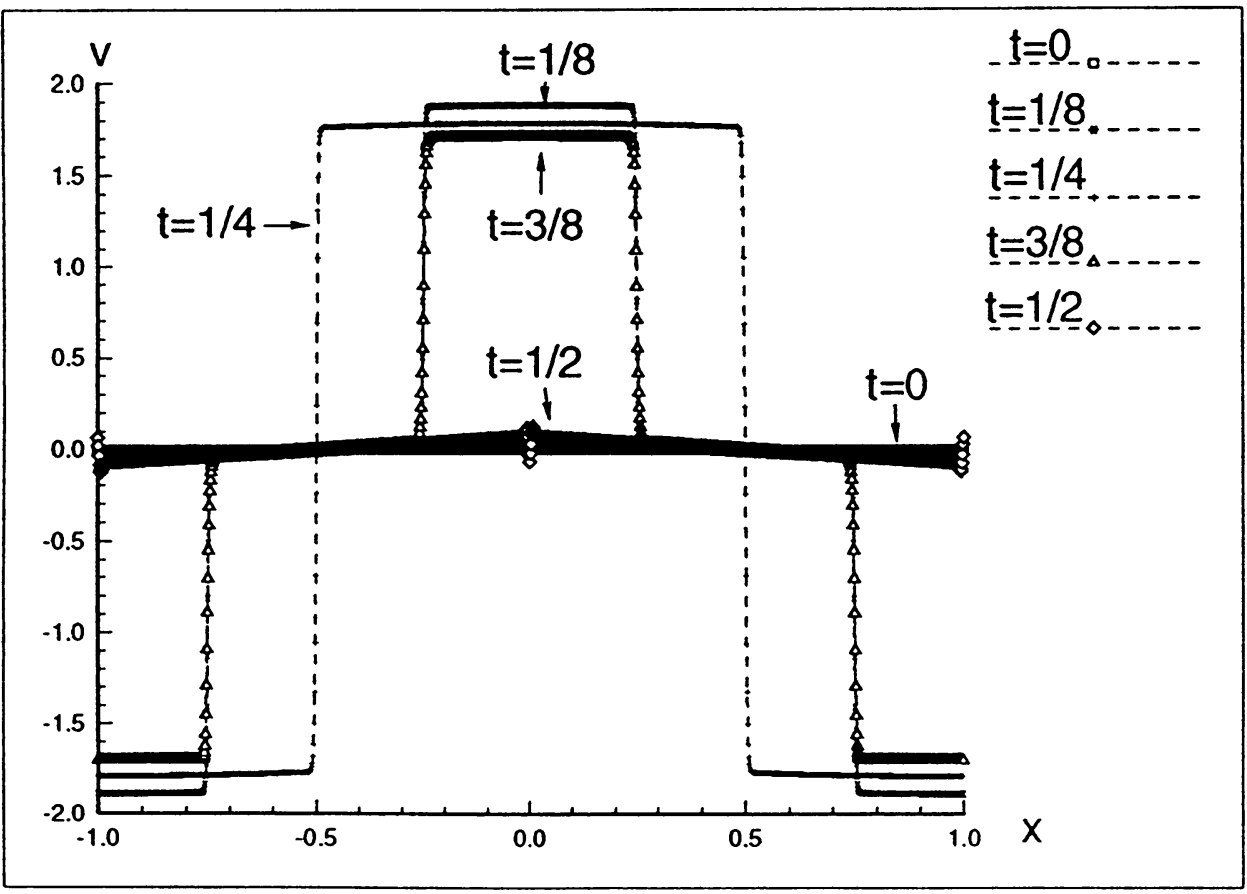

FIG. 3. The solution $v$ of Example 2, at $t=0, \frac{1}{8}, \frac{1}{4}, \frac{3}{8}$, and $\frac{1}{2}$.

results with 1600 mesh points are given in Figures 2 and 3. When we compare the curves in these two figures with the above given analytical results, we can see that all the numerical discontinuities have the right locations, and the strengths match (4.1) very well. We also see that $u$ is continuous when $t=\frac{1}{4}$, and $v$ is continuous when $t=\frac{1}{2}$. The numerical result for $t=\frac{1}{4}$ also matches (4.2) very well. The good agreement of the numerical results with the analytical results indicates that the scheme is reliable and robust for the resolution of discontinuities.

EXAMPLE 3. In this example we solve the nonlinear problem (1.1)-(1.3) with

$$
\begin{gathered}
\phi(u)=\psi(u)=2 u+5 u^{2}+25 u^{3}, \\
a(t)=0.4 e^{-t}+0.1 e^{-2 t}, \quad g(x, t)=0, \\
u_{0}(x)=2 \sin ^{3} x, \quad v_{0}(x)=2 \cos ^{3} x .
\end{gathered}
$$

We solve the problem numerically up to $t=0.5$ on the interval $[-\pi, \pi]$, imposing periodic boundary conditions. We again use zero threshold and take the factor as 1 . Numerical results with 1000 mesh points are given in Figures 4 and 5 (see p. 476). Notice that the initial data in this example are smooth and large. When $t=0.05$, the solution is still smooth. When $t=0.1$, we see that four numerical shocks have formed. Two of them are near the origin, while the other two are around the boundary. When $t=0.15$, these two pairs of shocks interact near the origin and the boundary. After interaction, the two shocks in each pair propagate in opposite directions; see the picture for $t=0.3$. At this 


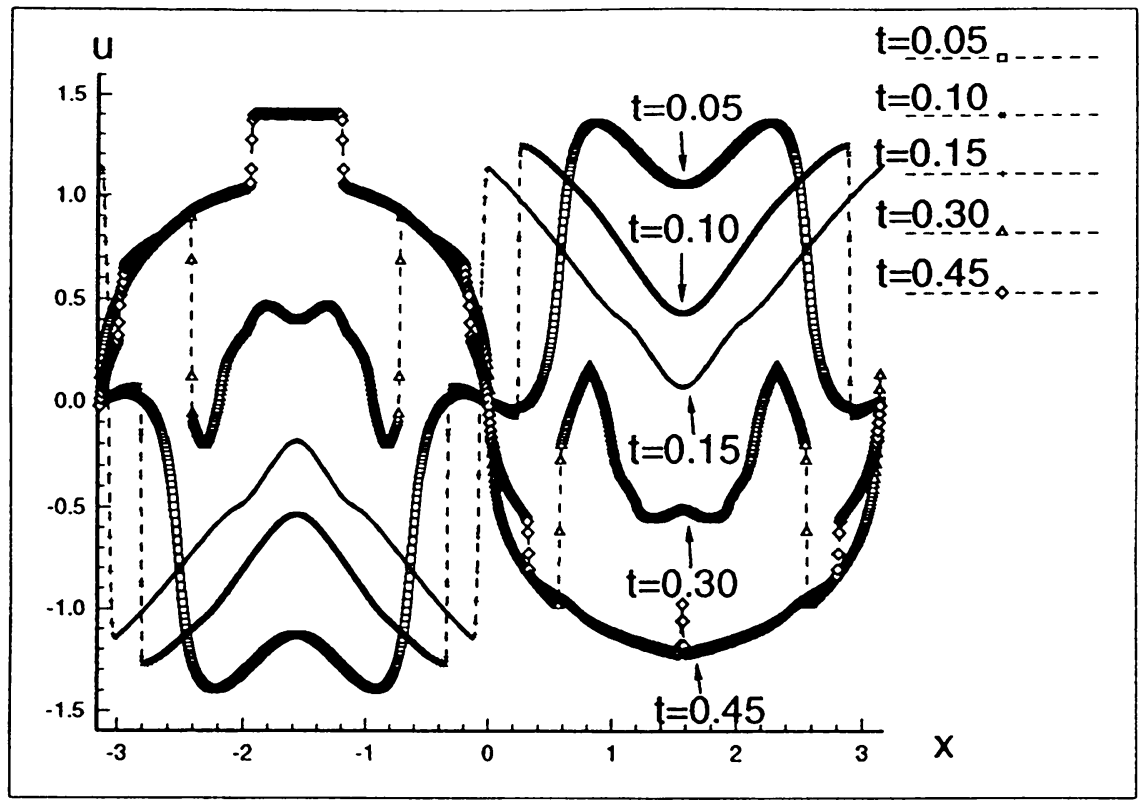

FIG. 4. The solution $u$ of Example 3, at $t=0.05,0.10,0.15,0.30$, and 0.45 .

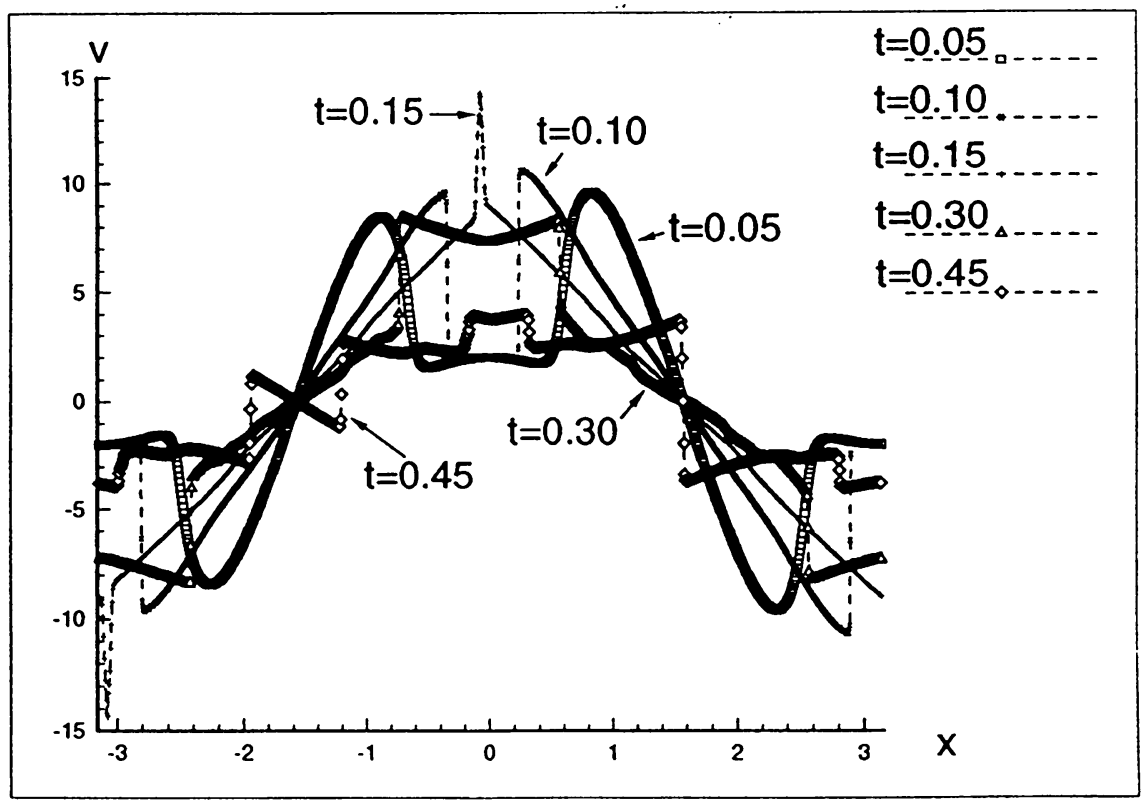

FIG. 5. The solution $v$ of Example 3, at $t=0.05,0.10,0.15,0.30$, and 0.45 . 
time there are also kinks (discontinuities in the derivative) in the solution. When $t=0.45$, we see that on the right-hand side of the origin, the two shocks interact near $x=1.57$, and two new shocks have formed near $x=0.32$ and $x=2.81$; while on the left-hand side of the origin, the two shocks have interacted and separated, and two new shocks are about to form near $x=-0.15$ and $x=-2.98$. This example shows that shocks may develop if the initial data are smooth but large. In [13] there is a similar example solved by the Lax-Wendroff scheme. Here, however, our scheme captures shocks much more sharply without oscillations, and the high resolution allows us to compute to a long time, hence finding shock interactions and the generation of more shocks. At this point we want to point out that there is no rigorous theoretical justification about the shocks in this example. However, considering that the scheme is stable and produced nice agreement with theory in the last example, there is a high fidelity in the numerical results.

EXAmple 4. This example is a Riemann problem. We consider (1.1)-(1.3) with

$$
\begin{gathered}
\phi(u)=\psi(u)=u+\frac{1}{3} u^{3}, \\
a(t)=0.4 e^{-t}+0.1 e^{-2 t}, \quad g(x, t)=0,
\end{gathered}
$$

and

$$
u_{0}(x)=\left\{\begin{array}{ll}
\frac{1}{2}, & x<0, \\
\frac{3}{2}, & x>0,
\end{array} \quad v_{0}(x)= \begin{cases}0, & x<0, \\
\frac{5}{\sqrt{24}}, & x>0 .\end{cases}\right.
$$

The Riemann data (4.4) consist of a 1-shock of the hyperbolic system

$$
\left\{\begin{aligned}
u_{t}-v_{x} & =0 \\
v_{t}-p(u)_{x} & =0
\end{aligned}\right.
$$

where

$$
p(u) \equiv \phi(u)-a(0) \psi(u)=\frac{1}{2}\left(u+\frac{1}{3} u^{3}\right) .
$$

The shock speed $c=-1.0206$ and the Riemann data satisfy the Rankine-Hugoniot condition and admissibility criterion (3.13). Moreover, the nonresonance condition (3.14) is also satisfied. From Sec. 3 we know that system (1.1), (1.3) possesses a smooth and strictly monotone traveling wave with speed $c$. It is our conjecture that this traveling wave is the asymptotic solution of our Riemann problem, where the translation is determined by the initial perturbation. As we see in Sec. 3, the local behavior of the Riemann problem is governed by the hyperbolic system

$$
\left\{\begin{aligned}
u_{t}-v_{x} & =0 \\
v_{t}-\phi(u)_{x} & =0
\end{aligned}\right.
$$

With $\phi$ given by (4.3), we see by solving the Riemann problem (4.7), (4.4) that for infinitesimal $t$, the initial discontinuity decomposes into two waves, a back shock with speed $s=-1.396$ and a front rarefaction with speeds $1.705 \leq x / t \leq 1.803$. Then as these two waves propagate, the strengths decay in $t$. Especially, if the asymptotic solution is indeed the aforementioned smooth traveling wave, the amplitude of the back shock must decay to zero and the speed $s$ must approach to $-\sqrt{\phi^{\prime}(0.5)}=-1.118$. 


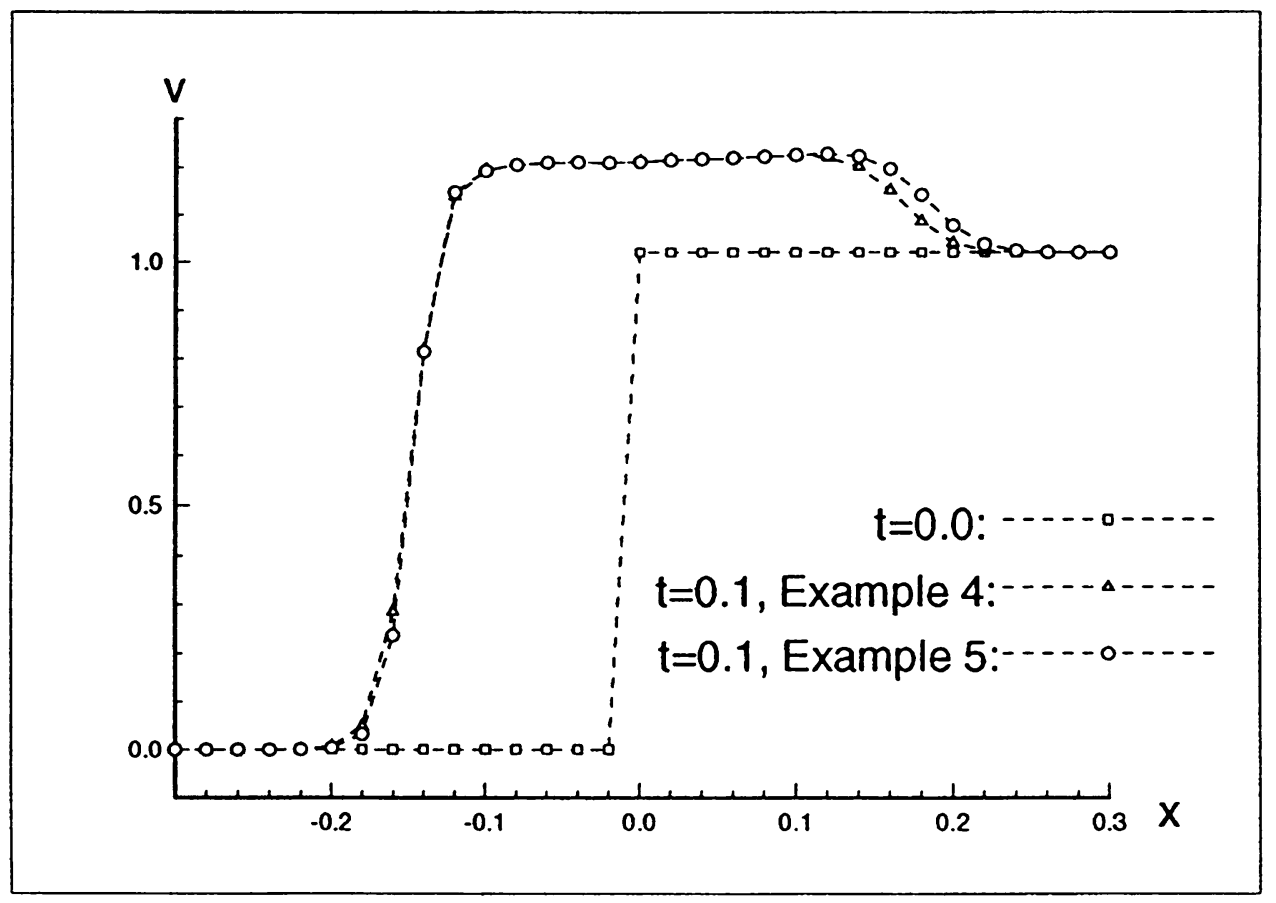

FIG. 6. The solution $v$. The initial condition $t=0$, and the result at $t=0.1$ for Example 4 and Example 5 .

We compute the solution up to $t=24$ on the interval $[-48,48]$, using 4800 grid points. The numerical boundary condition is taken as the Riemann data; again the threshold is zero and the factor is 1 . One of the curves in Fig. 6 is the result of component $v$ when $t=0.1$. We can see a back shock with speed around -1.39 and a front rarefaction-like wave, which is compatible with the aforementioned rarefaction for infinitesimal $t$.

The result for $u$ for $t$ up to 24 is given in Fig. 7; the result for $v$ is similar and hence not shown to save space.

Figure 8 is a blow-up of the rarefaction region of Fig. 7, where we can see clearly the initial rather rapid decay of the rarefaction-like wave. It seems that the decay slows down after a while, and it is not clear whether the solution will approach a monotone asymptotic solution.

Figure 9 (see p. 481) is a blow-up of the shock region in Fig. 7, where we can see clearly the decay of the shock. In fact, when $t=19.2$, the solution is practically continuous. It is easy to see from $(1.1),(1.3)$ that in front of the shock, the solution takes the constant state $\left(\frac{1}{2}, 0\right)$. Hence the shock locations are easily identified in Fig. 7. Comparing the curves for $t=21.6$ and $t=24$, a blow-up in the shock area (not shown to save space) shows that the shock speed $s$ approaches -1.118 indeed. From Fig. 7 , we see that the asymptotic solution is smooth if it exists. Again, comparing the curves for $t=21.6$ and $t=24$, we find that the wave center is moving with a speed of -1.02 , which is very close to the traveling wave speed $c$ mentioned above. All these show that the scheme handles shocks as expected. 


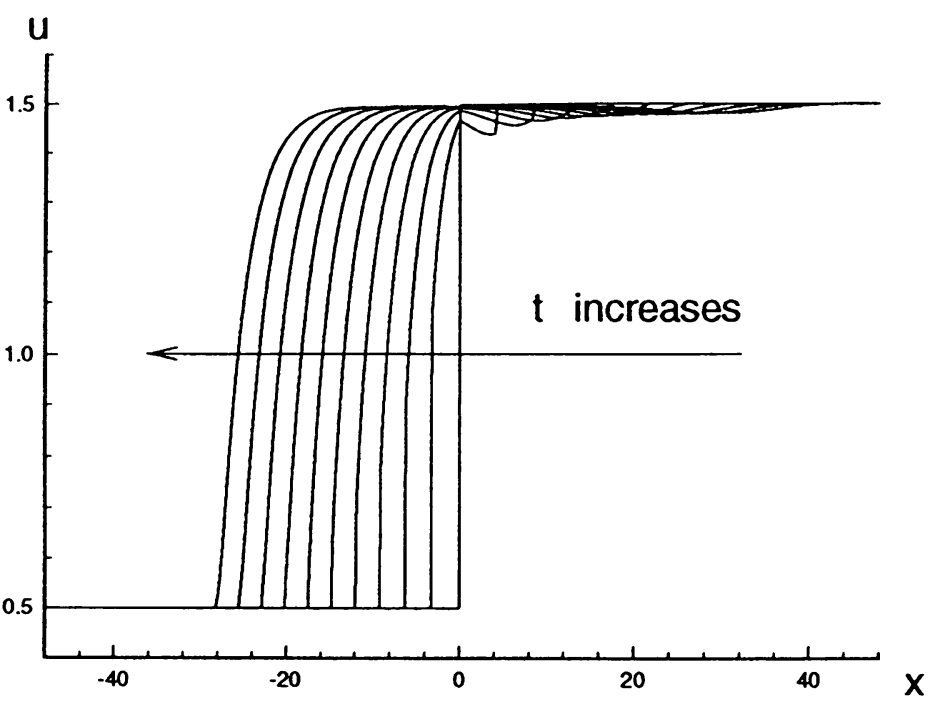

FIG. 7. The solution $u$ of Example 4, at $t=0.0,2.4,4.8,7.2,9.6$, $12.0,14.4,16.8,19.2,21.6$, and 24.0.

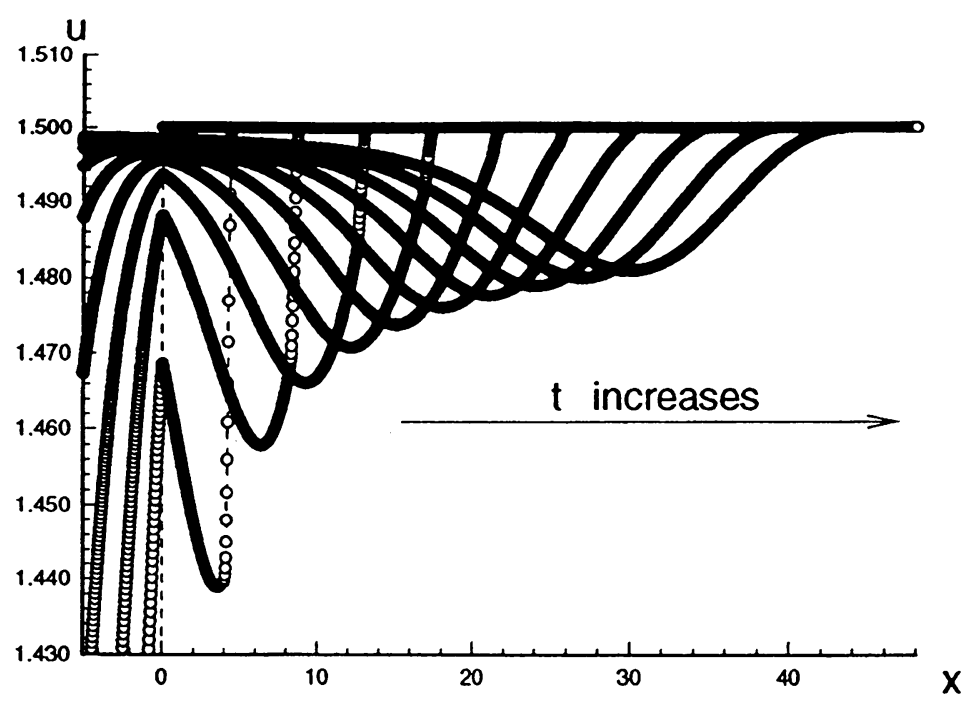

FIG. 8. The blow-up of Fig. 7, for the solution $u$, near the rarefaction region, of Example 4, at $t=0.0,2.4,4.8,7.2,9.6$, $12.0,14.4,16.8,19.2,21.6$, and 24.0 . 
EXAMPLE 5. This example is a Riemann problem with $\phi$ and $\psi$ different. We solve problem (1.1)-(1.3), where

$$
\begin{gathered}
\phi(u)=\frac{1}{2} u+\frac{1}{2} u^{3}, \quad \psi(u)=\frac{1}{3} u^{3}, \\
a(t)=0.5 e^{-t}+0.5 e^{-2 t}, \quad g(x, t)=0,
\end{gathered}
$$

and $u_{0}, v_{0}$ are given by (4.4). For such $\phi, \psi$, and $a$, the corresponding $p$ defined by (4.6) is exactly the same function as in Example 4. The Riemann data consist of a 1-shock of system (4.5). This time, however, the nonresonance condition (3.14) fails:

$$
c^{2}=1.0416>\phi^{\prime}\left(\frac{1}{2}\right)=0.875 \text {. }
$$

From Sec. 3, system (1.1), (1.3) has a monotone traveling wave with speed $c$ such that any discontinuity contained therein is a 1-shock of system (4.7) propagating with the same speed $c$. Again it is a conjecture that this traveling wave is the time-asymptotic solution of our Riemann problem, where the translation is determined by the initial perturbation. The local behavior of the solution is similar to that in Example 4: For infinitesimal $t$, there are a back shock with speed $s=-1.3909$ and a front rarefaction with speeds $1.8407 \leq x / t \leq 1.9685$.

In this example again we use zero threshold, take the factor as 1 and use the Riemann data as the numerical boundary condition. We first compare the local behavior with Example 4 by computing the solution for $t=0.1$, using the same interval and the same number of grid points. The result is given in Fig. 6. We see that the shock area almost overlaps the one of Example 4 since the two shock speeds are almost equal. The rarefaction-like wave, however, moves faster than that of Example 4, due to a greater speed. We then compute the solution up to $t=50$ on the interval $[-100,100]$ using 4800 grid points. The result of $u$ is given in Fig. 10. The result of $v$ is similar and hence not shown to save space.

Figure 11 (see p. 482) is a blow-up of the rarefaction region of Fig. 10, where we can see the decay of the rarefaction-like wave. Again, the decay seems to slow down after a while, and it is not clear whether the solution will approach a monotone asymptotic solution.

Figure 12 (see p. 482) is a blow-up of the shock area in Fig. 10. We see that the picture is different from Example 4: The shock strength decays at the beginning, and then tends to a limit. From a blow-up of the last two curves in Fig. 10 (not shown to save space), we find that the shock speed is about -1.03 for $45 \leq t \leq 50$, and the profile moves with a speed about -1.02 in this time period. Both speeds are very close to the traveling wave speed $c=-1.0206$ mentioned above. The nondecay of the shock may be explained as follows: at the beginning, the shock generated by the initial discontinuity tends to decay, which we know from the Green's function. As it propagates, the shock reduces speed. The traveling wave then catches up with the shock, and the hyperbolicity of the system comes to play, which may generate singularities; see Example 3. Notice that in Example 4 , the traveling wave never catches up with the shock. The numerical results in this 


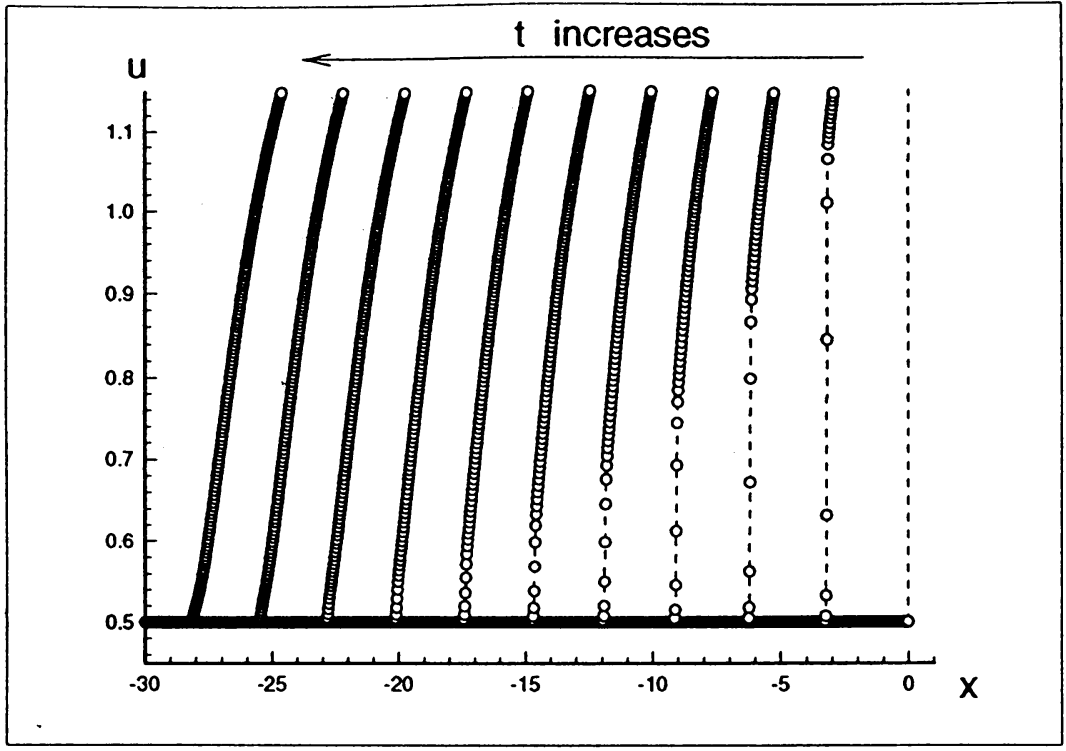

FIG. 9. The blow-up of Fig. 7, for the solution $u$, near the shock region, of Example 4, at $t=0.0,2.4,4.8,7.2,9.6,12.0$, 14.4, 16.8, 19.2, 21.6, and 24.0.

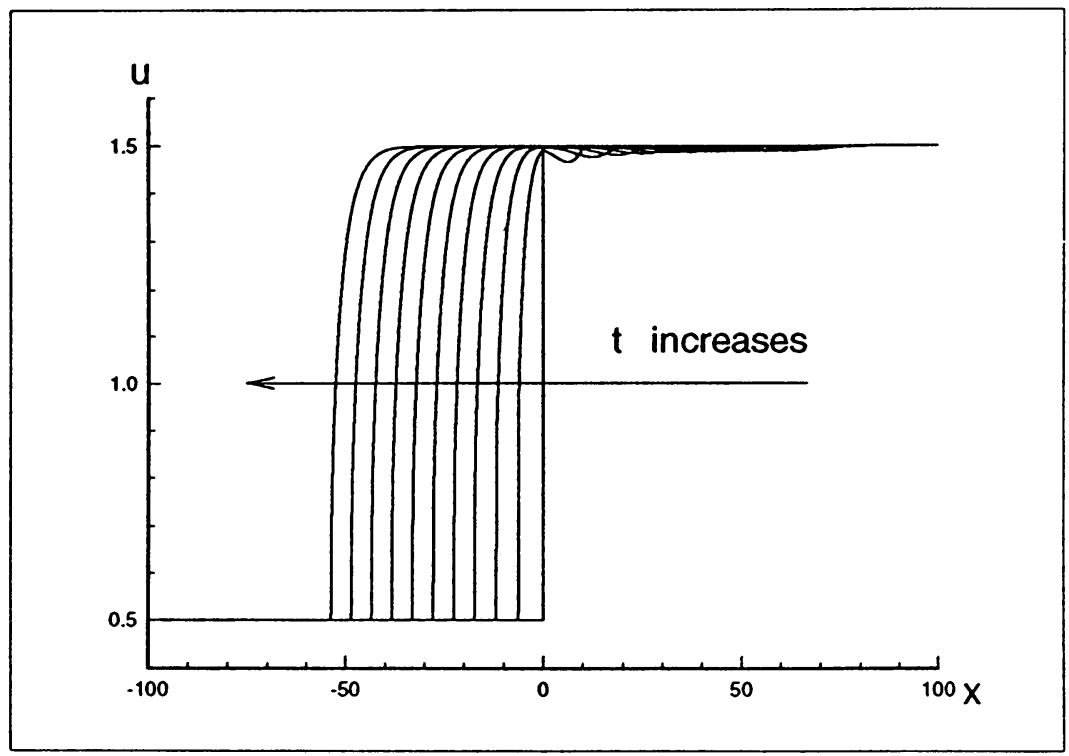

FIG. 10. The solution $u$ of Example 5 , at $t=0,5,10,15,20$, $25,30,35,40,45$, and 50 . 


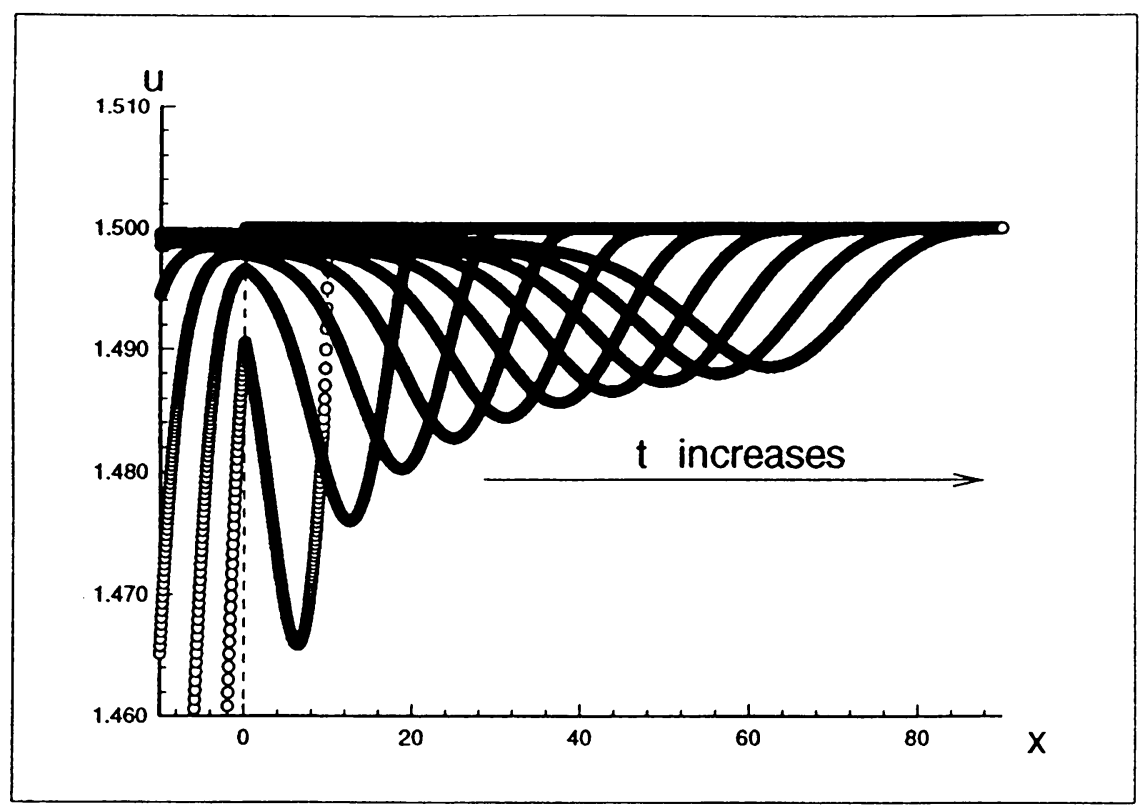

Fig. 11. The blow-up of Fig. 10, for the solution $u$, near the rarefaction region, of Example 5, at $t=0,5,10,15,20,25,30,35,40,45$, and 50 .

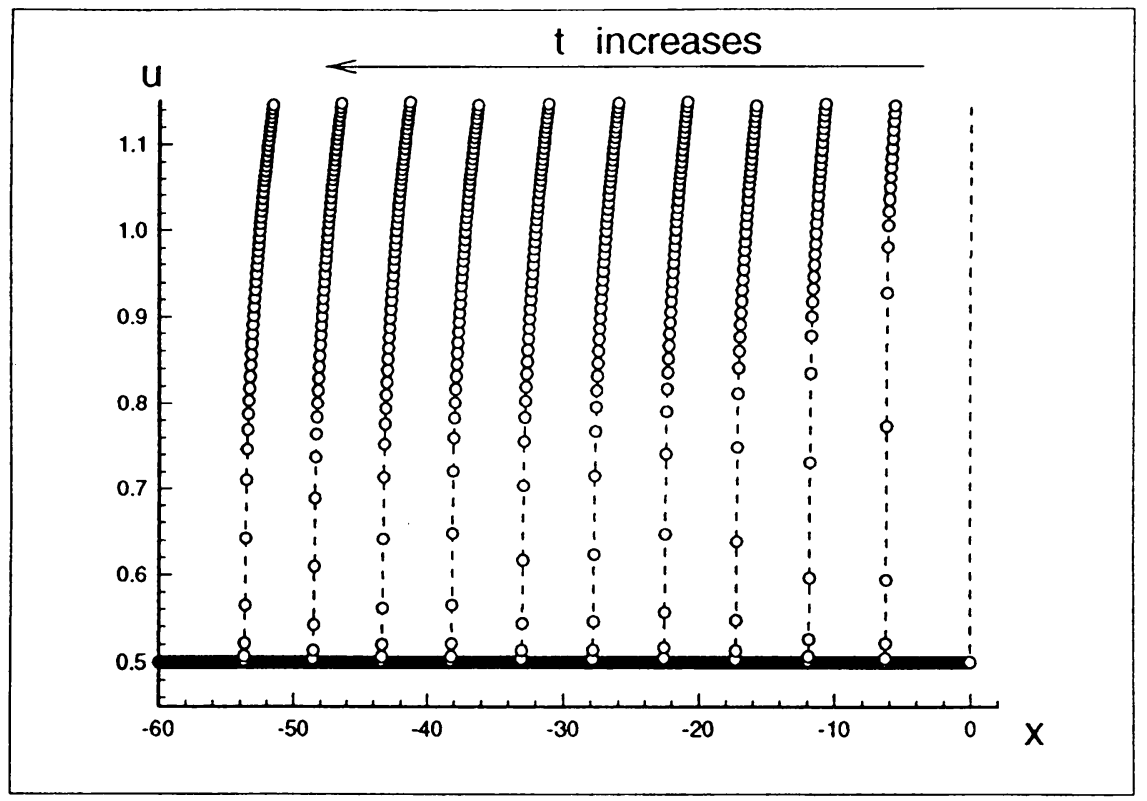

FIG. 12. The blow-up of Fig. 10: the solution $u$, near the shock region, of Example 5, at $t=0,5,10,15,20,25,30,35,40,45$, and 50 . 
example again match the theoretical prediction, and serve as a tool to verify theoretical conjectures, which again shows the usefulness of the scheme.

5. Concluding remarks. High-order ENO schemes are suitable for the numerical solution of viscoelasticity equations with fading memory. Computational results verify the theory about shock location and strength, and smooth region resolution, when such information is known. This gives us confidence about the reliability of the scheme. The scheme is then applied to nonlinear equations whose solution is not known. The scheme should provide a robust numerical tool to study such equations when rigorous analysis is not available, and it should provide a useful practical tool when numerical solutions for such type of equations are needed in applications.

\section{REFERENCES}

[1] J. Casper, C.-W. Shu, and H. Atkins, A comparison of two formulations for high-order accurate essentially non-oscillatory schemes, AIAA J. 32, 1970-1977 (1994)

[2] C. M. Dafermos, Development of singularities in the motion of materials with fading memory, Arch. Rational Mech. Anal. 91, 193-205 (1986)

[3] C. M. Dafermos and J. A. Nohel, Energy methods for nonlinear hyperbolic Volterra integrodifferential equations, Comm. Partial Differential Equations 4, 219-278 (1979)

[4] C. M. Dafermos and J. A. Nohel, A nonlinear hyperbolic Volterra equation in viscoelasticity, in Contributions to Analysis and Geometry, Amer. J. Math. Suppl., D. N. Clark, G. Pecelli and R. Sacksteder, eds., The Johns Hopkins University Press, Baltimore, London, 1981, pp. 87-116

[5] A. Harten, B. Engquist, S. Osher,and S. Chakravarthy, Uniformly high order essentially nonoscillatory schemes, III, J. Comput. Phys. 71, 231-303 (1987)

[6] W. J. Hrusa and J. A. Nohel, The Cauchy problem in one-dimensional nonlinear viscoelasticity, J. Differential Equations 59, 388-412 (1985)

[7] S. Kawashima, Large-time behavior of solutions to hyperbolic-parabolic systems of conservation laws and applications, Proc. Roy. Soc. Edinburgh 106A, 169-194 (1987)

[8] P. D. Lax, Development of singularities of solutions of nonlinear hyperbolic partial differential equations, J. Math. Phys. 5, 611-613 (1964)

[9] R. J. LeVeque, Numerical Methods for Conservation Laws, Birkhäuser-Verlag, Basel, 1990

[10] T.-P. Liu, Nonlinear waves for viscoelasticity with fading memory, J. Differential Equations 76, 26-46 (1988)

[11] T.-P. Liu and Y. Zeng, Large time behavior of solutions of general quasilinear hyperbolic-parabolic systems of conservation laws, Mem. Amer. Math. Soc., vol. 125, no. 599, 1997

[12] R. C. MacCamy, A model for one-dimensional nonlinear viscoelasticity, Quart. Appl. Math. 35, 21-33 (1977)

[13] P. Markowich and M. Renardy, Lax-Wendroff methods for hyperbolic history value problems, SIAM J. Numer. Anal. 21, 24-51 (1984). Errata in 22, 204 (1985)

[14] J. A. Nohel and M. Renardy, Development of singularities in nonlinear viscoelasticity, in Amorphous Polymers and Non-Newtonian Fluids, C. Dafermos, J. L. Ericksen, and D. Kinderlehrer, eds., Springer, New York, 1987, pp. 139-152

[15] J. A. Nohel, R. C. Rogers, and A. E. Tzavaras, Weak solutions for a nonlinear system in viscoelasticity, Comm. Partial Differential Equations 13, 97-127 (1988)

[16] C.-W. Shu and S. Osher, Efficient implementation of essentially non-oscillatory shock capturing schemes, J. Comput. Phys. 77, 439-471 (1988)

[17] C.-W. Shu and S. Osher, Efficient implementation of essentially non-oscillatory shock capturing schemes II, J. Comput. Phys. 83, 32-78 (1989)

[18] C.-W. Shu, Numerical experiments on the accuracy of ENO and modified ENO schemes, J. Sci. Comput. 5, 127-149 (1990)

[19] C.-W. Shu, T. A. Zang, G. Erlebacher, D. Whitaker, and S. Osher, High order ENO schemes applied to two and three dimensional compressible flow, Appl. Numer. Math. 9, 45-71 (1992)

[20] O. Staffans, On a nonlinear hyperbolic Volterra equation, SIAM J. Math. Anal. 11, 793-812 (1980) 
[21] Y. Zeng, Convergence to diffusion waves of solutions to nonlinear viscoelastic model with fading memory, Comm. Math. Phys. 146, 585-609 (1992)

[22] Y. Zeng, $L^{1}$ asymptotic behavior of compressible, isentropic, viscous 1-D flow, Comm. Pure Appl. Math. 47, 1053-1082 (1994)

[23] Y. Zeng, $L^{p}$ asymptotic behavior of solutions to hyperbolic-parabolic systems of conservation laws, Arch. Math. 66, 310-319 (1996) 